\title{
UNIFIED STABILIZED FINITE ELEMENT FORMULATIONS FOR THE STOKES AND THE DARCY PROBLEMS*
}

\author{
SANTIAGO BADIA ${ }^{\dagger}$ AND RAMON CODINA R $^{\ddagger}$
}

\begin{abstract}
In this paper we propose stabilized finite element methods for both Stokes' and Darcy's problems that accommodate any interpolation of velocities and pressures. Apart from the interest of this fact, the important issue is that we are able to deal with both problems at the same time, in a completely unified manner, in spite of the fact that the functional setting is different. Concerning the stabilization formulation, we discuss the effect of the choice of the length scale appearing in the expression of the stabilization parameters, both in what refers to stability and to accuracy. This choice is shown to be crucial in the case of Darcy's problem. As an additional feature of this work, we treat two types of stabilized formulations, showing that they have a very similar behavior.
\end{abstract}

Key words. Stokes-Darcy's problem, stabilized finite element methods, characteristic length scale, orthogonal subgrid scales

AMS subject classifications. 65N30, 35Q30

DOI. $10.1137 / 08072632 \mathrm{X}$

1. Introduction. In this paper we present a unified finite element approximation of the Stokes and the Darcy problems, which allows the use of arbitrary conforming velocity-pressure interpolations. The key ingredient in the unified treatment of both problems is to consider a generalized Stokes problem with a source term that we will call Stokes-Darcy problem (and which is also called the Brinkman model), and define a functional setting that is well behaved both when the viscosity is zero (Darcy's problem) and when there is no source term (Stokes' problem). This is possible not only for the discrete finite element problem, but also at the space continuous level. The analysis of the continuous case provides a valuable guide about the results to be expected in the approximated problem. The graph norm for the diffusion-reaction operator associated to the velocity allows us to construct a norm in the velocity-pressure space that has the correct behavior in the limits $\nu \rightarrow 0$ (zero viscosity, that is to say, Darcy's problem) and $\sigma \rightarrow 0$ (infinite permeability, that is to say, the classical Stokes problem).

The Galerkin approximation of both the Stokes and the Darcy problems requires the use of velocity-pressure interpolations that satisfy the adequate inf-sup conditions (see, for example, $[6,19]$ ). Different interpolation pairs are known to satisfy this condition for each problem independently, but the key issue is to find interpolations that satisfy both at the same time. The design of this kind of mixed velocity-pressure interpolations, even in the nonconforming case for the Stokes problem, is a difficult

\footnotetext{
${ }^{*}$ Received by the editors June 5, 2008; accepted for publication (in revised form) February 11, 2009; published electronically May 27, 2009.

http://www.siam.org/journals/sinum/47-3/72632.html

$\dagger$ International Center for Numerical Methods in Engineering (CIMNE), Universitat Politècnica de Catalunya, Jordi Girona 1-3, Edifici C1, 08034 Barcelona, Spain (sbadia@cimne.upc.edu). Current address: Applied Mathematics and Applications, MS-1320, Sandia National Laboratories, Albuquerque, NM 87185-1320. This author's research was supported by the European Community through the Marie Curie contract NanoSim (MOIF-CT-2006-039522).

¥International Center for Numerical Methods in Engineering (CIMNE), Universitat Politècnica de Catalunya, Jordi Girona 1-3, Edifici C1, 08034 Barcelona, Spain (ramon.codina@upc.edu).
}

1971 
task (see $[24,1])$. Furthermore, the resulting mixed interpolations are expensive and in some cases restricted to specific typologies of meshes.

In order to alleviate this restriction, we choose here the use of stabilized finite element methods. In fact, we will show that with the formulations we analyze, it is possible to use any velocity-pressure pair, provided the discrete spaces are conforming. The most important feature, however, is that the same formulation works for the Stokes and the Darcy problems.

The framework of our stabilization techniques is the variational multiscale method, proposed in the finite element context in [20,21]. It is based on the decomposition of the unknowns into their finite element component and a subscale, that is, the component of the continuous unknown that cannot be captured by the finite element mesh. However, we will not describe here neither the motivation of the formulations nor the design of the stabilization parameters on which they depend. Our concern is to analyze the convergence properties of the formulations and to show that they are optimal in both the Stokes and the Darcy limits. We will see that the continuous functional setting is reproduced by the stabilized finite element methods we analyze. We consider two possibilities, namely, what we call an algebraic subgrid scale (ASGS) approach and the orthogonal subscale stabilization (OSS) method.

An important aspect of the work presented is that we discuss the effect of the

choice of the length scale appearing in the expression of the stabilization parameters, both in what refers to stability and to accuracy. This choice is shown to be crucial in the case of Darcy's proble and pressure interpolatio The literature about the Darcy problems is the stabilization technic $[26,22])$. It is what we will
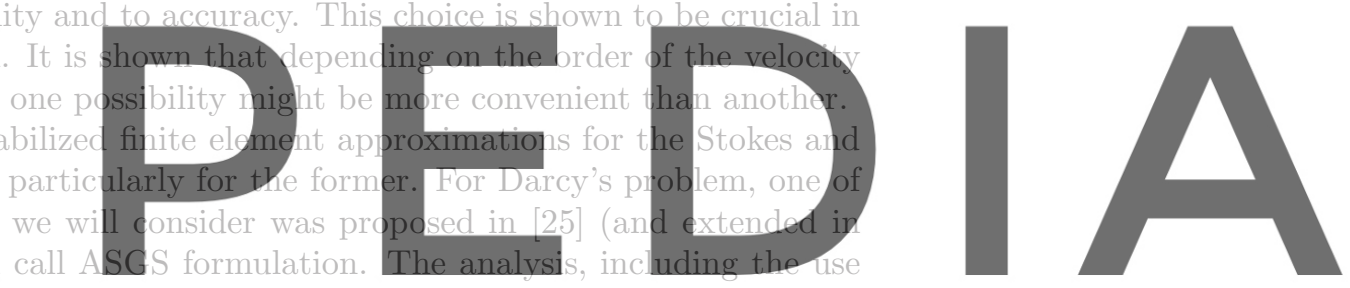

of discontinuous velocity interpolations, can be found in [7]. The second formulation,

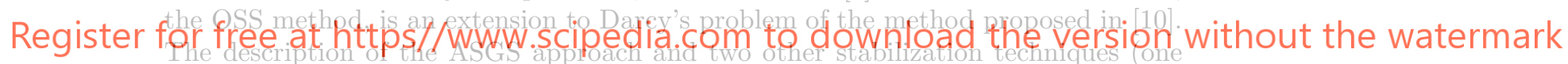

of them originally designed for the Stokes problem in [18]) can be found in [4]. An alternative to the use of stabilization techniques for Darcy's problem is to use leastsquare formulations, which can also be used together with interpolations satisfying the inf-sup condition (see, for example, [5]).

For both the ASGS and the OSS formulations, it is crucial to introduce pressure jumps across interelement boundaries to allow the use of discontinuous pressure interpolations. The motivation of our way to deal with these jumps can be found in [15]. Similar ideas have been used in [14] applied to the three field formulation of the Stokes problem.

Even though we will pay attention to the Darcy problem, our starting objective is to deal with the Stokes-Darcy problem. The ASGS formulation allows us to do that, and, in fact, a complete analysis was already undertaken in [11]. However, the design of the stabilization parameters in that reference does not allow us to consider $\nu=0$, that is, the pure Darcy problem. Several other attempts based on some sort of stabilization can be found in the literature. For example, in [9] it is proposed to use a continuous linear velocity and piecewise constant pressure interpolation, since a common stabilization procedure can be designed for the Stokes and the Darcy problems using this element (even though these two problems are studied independently).

Another reason of interest for the analysis of the Stokes and the Darcy problems is the coupling of both through interfaces, possibly using domain decomposition 
strategies. A way to deal with this problem is succinctly explained in section 5 , and a numerical example is presented in section 6 . As it is indicated in section 5, our unified formulation for both problems naturally motivates a monolithic approach to this coupled problem. For the time being, let us mention that the choice of the approximation spaces in each subproblem is crucial in the domain decomposition algorithm (see, for example, $[17,16,23,27])$. The possibility of using the same interpolation for each subproblem offered by our stabilized formulations clearly simplifies the enforcement of the transmission conditions on the interfaces.

The paper is organized as follows. In section 2 we state the continuous problem and prove and inf-sup condition, which clearly displays the functional setting of the problem. This analysis is valid for all values of viscosity and inverse of permeability, including the case when one of these two parameters is zero. The analysis of the stabilized formulations we propose is presented in section 3. We describe the stabilized methods we wish to consider and prove optimal stability and convergence results for each. These formulations depend on algorithmic parameters, the so-called stabilization parameters, which, in turn, depend on a length scale of the problem that needs to be chosen a priori. Whereas this length scale is irrelevant when there is viscosity, it plays a crucial role in the accuracy of the stabilized Darcy formulation. To highlight this effect, in section 4 we obtain $L^{2}$-error estimates for the velocity and the pressure using duality arguments for the Darcy problem. Section 5 is devoted to explain how the coupling of Stokes and Darcy problems through interfaces can be done. The rates of convergence predicted by the theory are confirmed in the numerical tests carriod
out in section 6 . Some concluding rernarks close the paper in section 7 .
2. Continuous problem.
2.1. Problem statement. I et $\Omega \subset \mathbb{R}^{d}, d=2,3$, be a polyhedral domain (With
Lipschitz boundary) where we consiler the Stokes Darcy (or Brinknan) problem, which consists in finding a velocity $u: \Omega \longrightarrow \mathbb{R}^{d}$ and a pressure $p: \Omega \longrightarrow \mathbb{R}$ such that

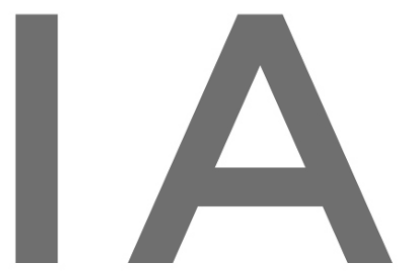

\section{Register fonfree at https//www.scipedia،coma to download the version without the watermark $(2.2)$ $\nabla \cdot u=g$,}

where $\boldsymbol{f}$ and $g$ are given data and the physical parameters are the viscosity $\nu$ and the inverse of the permeability $\sigma$. For simplicity, as boundary conditions we will consider $\boldsymbol{u}=\mathbf{0}$ if $\nu>0$ and $\boldsymbol{n} \cdot \boldsymbol{u}=0$ if $\nu=0, \boldsymbol{n}$ being the unit normal exterior to $\partial \Omega$. In the second case we could also consider the pressure prescribed on part of the boundary, although this would not affect significantly the following developments. In all what follows we will consider that either $\nu$ or $\sigma$ may be zero, but obviously not both.

Let $H_{0}^{1}(\Omega)^{d}$ be the space of vector-valued functions with components in $H^{1}(\Omega)$ with zero trace on $\partial \Omega$, with dual $H^{-1}(\Omega)^{d}$, and let $H_{0}(\operatorname{div}, \Omega)$ be the space of vector fields in $H(\operatorname{div}, \Omega)$, with zero normal trace on $\partial \Omega$. If $\nu>0$, the problem is well posed if the unknowns are taken as $\boldsymbol{u} \in H_{0}^{1}(\Omega)^{d}, p \in L^{2}(\Omega)$, and the data are such that $f \in H^{-1}(\Omega)^{d}, g \in L^{2}(\Omega)$. If $\nu=0$, that is, for the Darcy problem, the problem can be thought in two different ways:

1. The singular limit $\nu \rightarrow 0$. In this case it would be natural to require that

$$
\boldsymbol{u} \in H_{0}(\operatorname{div}, \Omega), \quad p \in L^{2}(\Omega) / \mathbb{R}, \quad \boldsymbol{f} \in H_{0}(\operatorname{div}, \Omega)^{\prime}, \quad g \in L^{2}(\Omega) .
$$

The limit $\nu \rightarrow 0$ is singular in the sense that the regularity of the velocity drops from $H^{1}(\Omega)^{d}$ to $H(\operatorname{div}, \Omega)$, but the regularity of the pressure is maintained. The regularity required for the data is modified accordingly. 
2. A mixed formulation of the Poisson problem. In this case, the functional setting is

$$
\boldsymbol{u} \in L^{2}(\Omega)^{d}, \quad p \in H^{1}(\Omega) / \mathbb{R}, \quad \boldsymbol{f} \in L^{2}(\Omega)^{d}, \quad g \in H^{-1}(\Omega) .
$$

We may take $\boldsymbol{f}=\mathbf{0}$. Otherwise, we may decompose $\boldsymbol{f}=\nabla p_{0}+\boldsymbol{u}_{0}$, with $\nabla \cdot \boldsymbol{u}_{0}=0$, and take as unknowns $\boldsymbol{u}-\boldsymbol{u}_{0}$ and $p-p_{0}$. Note that the boundary condition $\boldsymbol{n} \cdot \boldsymbol{u}=0$ holds in $H^{-1 / 2}(\partial \Omega)$. In this case it would be convenient to prescribe boundary conditions on the pressure, but, as it has been mentioned, this is irrelevant for our discussion.

In fact, whichever the situation is, it will be determined by the data. In the next subsection we will obtain an inf-sup condition that can be trivially translated into velocity-pressure stability if the data are regular enough.

Let us denote by $\left\langle f_{1}, f_{2}\right\rangle$ the integral of two (generalized) functions $f_{1}$ and $f_{2}$ (either scalar or vector-valued) in $\Omega$. The regularity of both is such that the integral is well defined. For example, if $f_{1} \in H_{0}^{1}(\Omega)$, we may take $f_{2} \in H^{-1}(\Omega)$. When both $f_{1}, f_{2} \in L^{2}(\Omega)$, we will write their $L^{2}(\Omega)$ inner product as $\left\langle f_{1}, f_{2}\right\rangle \equiv\left(f_{1}, f_{2}\right)$. The associated norm will be denoted by $\left\|f_{1}\right\|_{L^{2}(\Omega)} \equiv\left\|f_{1}\right\|$. We will use $\|\cdot\|_{X}$ to indicate the norm in a Banach space $X$, with the abbreviation $\|\cdot\|_{-1}$ when $X=H^{-1}(\Omega)$.

Either in the situation (2.3) or in (2.4) the variational formulation of the problem consists in finding a velocity-pressure pair $[u, p]$ in the appropriate functional space

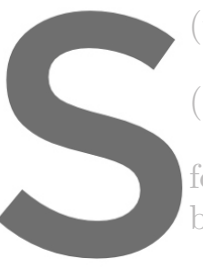
(to be specified in the fo

$(2.5)$ or all test functions $[v$,
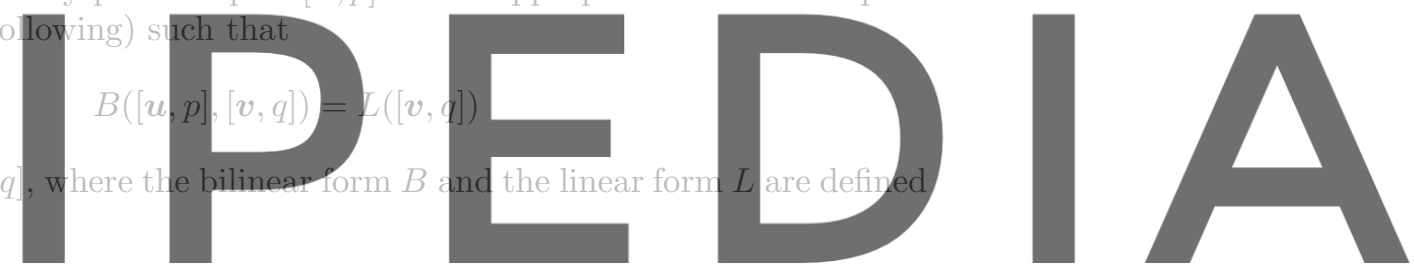

$(2.6)$

$$
B([u, p],[v, q])=\nu(\nabla u, \nabla v)+\sigma(u, v)-(p, \nabla \cdot v)+(q, \nabla \cdot u),
$$

\section{Register for free at https $\%$ wWwW.scipedial:com to download the version without the watermark}

The correct functional setting of the problem for all values of $\nu$ and $\sigma$ is a consequence of the inf-sup condition proved next.

2.2. Generalized stability estimate. Let us introduce the operator

$$
\mathcal{L} \boldsymbol{u}:=-\nu \Delta \boldsymbol{u}+\sigma \boldsymbol{u}
$$

and the associated graph norm

$$
\|\boldsymbol{u}\|_{\mathcal{L}}^{2}:=\nu\|\nabla \boldsymbol{u}\|^{2}+\sigma\|\boldsymbol{u}\|^{2} .
$$

Let $V_{\mathcal{L}}$ be the Banach space obtained as the closure of $C_{0}^{\infty}(\Omega)^{d}$ with respect to this norm. Its dual space $V_{\mathcal{L}}^{\prime}$ is endowed with the norm

$$
\|\boldsymbol{u}\|_{\mathcal{L}^{\prime}}:=\sup _{\boldsymbol{v} \in V_{\mathcal{L}}} \frac{\langle\boldsymbol{u}, \boldsymbol{v}\rangle}{\|\boldsymbol{v}\|_{\mathcal{L}}}
$$

Obviously, $V_{\mathcal{L}}=H_{0}^{1}(\Omega)^{d}, V_{\mathcal{L}}^{\prime}=H^{-1}(\Omega)^{d}$ if $\nu>0$, and $V_{\mathcal{L}}=V_{\mathcal{L}}^{\prime}=L^{2}(\Omega)^{d}$ if $\nu=0$.

A key ingredient in the following discussion is the introduction of a characteristic length scale of the problem that we denote by $L_{0}$, which may be taken, for example, as the diameter of the computational domain $\Omega$. Whereas for the Stokes problem, 
its introduction is unnecessary, it will play a key role in the Darcy problem. The ultimate reason to explain this fact is that in the Stokes case the seminorm $\|\nabla \boldsymbol{u}\|$ controls the whole norm in $H_{0}^{1}(\Omega)^{d}$ because of the Poincaré-Friedrichs inequality, and thus a stability estimate in this seminorm suffices. However, for the Darcy problem, we need to control both $\boldsymbol{u}$ and $\nabla \cdot \boldsymbol{u}$ to obtain stability in $H(\operatorname{div}, \Omega)$, and the only way to incorporate both norms in a single one is through the introduction of a length scale. While this discussion might seem unnecessary to obtain theoretical stability estimates (and thus to determine the functional framework of the problem), it will lead to important consequences in the discrete finite element problem.

Let now $V$ be the closure of $C_{0}^{\infty}(\Omega)^{d}$, with respect to the norm $\|\boldsymbol{v}\|_{\mathcal{L}}+\sqrt{\sigma} L_{0}\|\nabla \cdot \boldsymbol{v}\|$ and $Q$ the closure of $C^{\infty}(\Omega) / \mathbb{R}$, with respect to $\left(\nu+\sigma L_{0}^{2}\right)^{-1 / 2}\|q\|+\|\nabla q\|_{\mathcal{L}^{\prime}}$. The pair $V \times Q$ reduces to $H_{0}^{1}(\Omega)^{d} \times L^{2}(\Omega) / \mathbb{R}$ when $\nu>0$ and to $H_{0}(\operatorname{div}, \Omega) \times H^{1}(\Omega) / \mathbb{R}$ when $\nu=0$. On $V \times Q$ we define

\section{(2.10) $\quad\|[\boldsymbol{v}, q]\|^{2}:=\|\boldsymbol{v}\|_{\mathcal{L}}^{2}+\sigma L_{0}^{2}\|\nabla \cdot v\|^{2}+\frac{1}{\nu+\sigma L_{0}^{2}}\|q\|^{2}+\|\nabla q\|_{\mathcal{L}^{\prime}}^{2}$.}

Let us anticipate that this will be the finest norm in which stability can be proved without using shift assumptions that involve the regularity of the domain (see Remark 2.1 below).

In what follows, $C$ denotes a positive constant, in our case independent of $\nu$, $\sigma$, and $L_{0}$. When dealing with the finite element approximated problem, it will be occurrences. We will and $A \leq C B$, respective that in the discrete case THEOREM 2.1 (stabilit
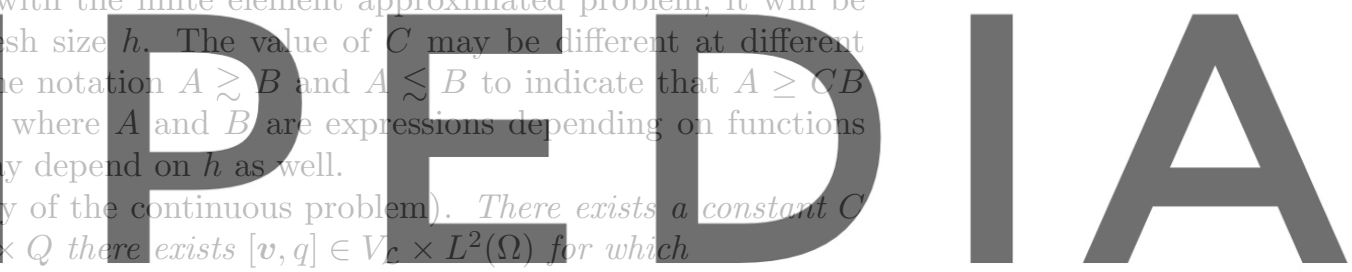

\section{$B([u, p],[v, q]) \geq C\|[u, p]\|\|[v, q]\|_{V_{C} \times L^{2}(\Omega)}$}

Register for free at https/ www ws sipedia. Com to download the version without the watermark Proof. Let $[u, p]$ be given. For $\left[v_{1}, q_{1}\right]=[u, p]$,

$$
B\left([\boldsymbol{u}, p],\left[\boldsymbol{v}_{1}, q_{1}\right]\right)=\|\boldsymbol{u}\|_{\mathcal{L}}^{2} .
$$

Because the divergence operator is onto both from $H_{0}^{1}(\Omega)^{d}$ and from $H_{0}(\operatorname{div}, \Omega)$ to $L^{2}(\Omega)$, we have the inf-sup conditions:

$$
\forall p \in L^{2}(\Omega) \text { there exist } \boldsymbol{v}_{p} \in H_{0}^{1}(\Omega)^{d} \mid-\left(p, \nabla \cdot \boldsymbol{v}_{p}\right) \gtrsim\|p\|\left\|\nabla \boldsymbol{v}_{p}\right\|,
$$

$\forall p \in L^{2}(\Omega)$ there exist $\boldsymbol{v}_{p} \in H_{0}(\operatorname{div}, \Omega) \mid-\left(p, \nabla \cdot \boldsymbol{v}_{p}\right) \gtrsim\|p\|\left(\frac{1}{L_{0}}\left\|\boldsymbol{v}_{p}\right\|+\left\|\nabla \cdot \boldsymbol{v}_{p}\right\|\right)$.

In the first case we have $\left\|\nabla \boldsymbol{v}_{p}\right\| \gtrsim\left(\frac{1}{L_{0}}\left\|\boldsymbol{v}_{p}\right\|+\left\|\nabla \cdot \boldsymbol{v}_{p}\right\|\right)$ (using Poincaré-Friedrichs's inequality), so that for the spaces $V$ and $Q$ we have defined, we have that for all $p \in Q$, there exists $\boldsymbol{v}_{p} \in V$ such that

$$
-\left(p, \nabla \cdot \boldsymbol{v}_{p}\right) \gtrsim\|p\| \frac{1}{\sqrt{\nu+\sigma L_{0}^{2}}}\left(\sqrt{\nu}\left\|\nabla \boldsymbol{v}_{p}\right\|+\sqrt{\sigma}\left\|\boldsymbol{v}_{p}\right\|+\sqrt{\sigma} L_{0}\left\|\nabla \cdot \boldsymbol{v}_{p}\right\|\right) .
$$

We may choose $\boldsymbol{v}_{p}$ such that

$$
\sqrt{\nu}\left\|\nabla \boldsymbol{v}_{p}\right\|+\sqrt{\sigma}\left\|\boldsymbol{v}_{p}\right\|+\sqrt{\sigma} L_{0}\left\|\nabla \cdot \boldsymbol{v}_{p}\right\|=\frac{1}{\sqrt{\nu+\sigma L_{0}^{2}}}\|p\|
$$


which is dimensionally consistent. For $\left[\boldsymbol{v}_{2}, q_{2}\right]=\left[\boldsymbol{v}_{p}, 0\right]$,

$$
\begin{aligned}
& B\left([\boldsymbol{u}, p],\left[\boldsymbol{v}_{2}, q_{2}\right]\right) \gtrsim-\nu\|\nabla \boldsymbol{u}\|\left\|\nabla \boldsymbol{v}_{p}\right\|-\sigma\|\boldsymbol{u}\|\left\|\boldsymbol{v}_{p}\right\|+\frac{1}{\nu+\sigma L_{0}^{2}}\|p\|^{2} \\
& \gtrsim-\|\boldsymbol{u}\|_{\mathcal{L}}\left\|\boldsymbol{v}_{p}\right\|_{\mathcal{L}}+\frac{1}{\nu+\sigma L_{0}^{2}}\|p\|^{2} \\
& \gtrsim-\|\boldsymbol{u}\|_{\mathcal{L}} \frac{1}{\sqrt{\nu+\sigma L_{0}^{2}}}\|p\|+\frac{1}{\nu+\sigma L_{0}^{2}}\|p\|^{2} \\
& \gtrsim-\|\boldsymbol{u}\|_{\mathcal{L}}^{2}+\frac{1}{\nu+\sigma L_{0}^{2}}\|p\|^{2}
\end{aligned}
$$

$(2.13)$

$$
B\left([u, p],\left[v_{3}, q_{3}\right]\right)=\sigma L_{0}^{2}\|\nabla \cdot u\|^{2} .
$$

Let now $\boldsymbol{v}_{g} \in V_{\mathcal{L}}$ be such that

$$
\|\nabla p\|_{\mathcal{L}^{\prime}}=\frac{\left\langle\nabla p, v_{g}\right\rangle}{\left\|v_{g}\right\|_{\mathcal{L}}}
$$

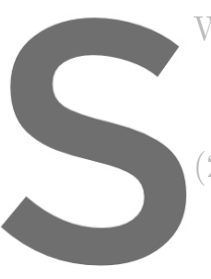

We may choose rg sirch
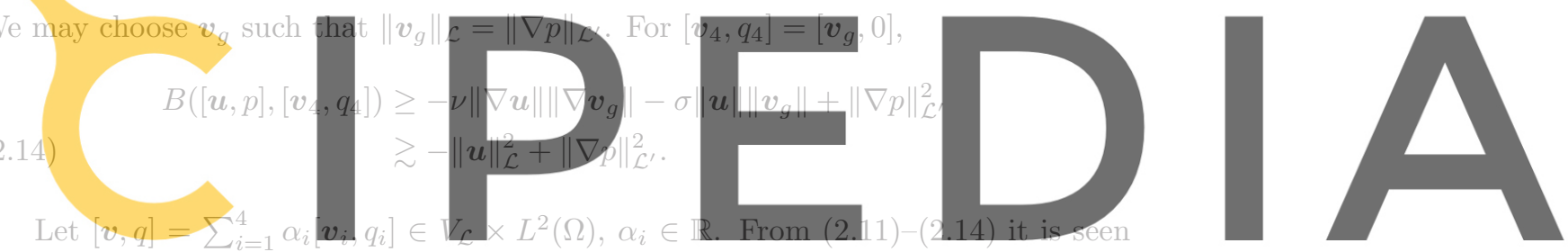

that the coefficients $\alpha_{i}$ can be chosen so that

\section{Register for free at https//wwwscipedia.com to download the version without the watermark}

The theorem follows noting that for any combination of coefficients $\alpha_{i} \in \mathbb{R}$, we have $\|[\boldsymbol{v}, q]\|_{V_{\mathcal{L}} \times L^{2}(\Omega)} \lesssim\|[\boldsymbol{u}, p]\|$.

Remark 2.1.

1. The working norm is optimal. Observe that

$$
\begin{array}{lrl}
\|[\boldsymbol{v}, q]\|^{2}=\nu\|\nabla \boldsymbol{u}\|^{2}+\frac{1}{\nu}\|p\|^{2}+\frac{1}{\nu}\|\nabla p\|_{-1}^{2} & \text { when } \sigma=0, \\
\|[\boldsymbol{v}, q]\|^{2}=\sigma\|\boldsymbol{u}\|^{2}+\sigma L_{0}^{2}\|\nabla \cdot \boldsymbol{u}\|^{2}+\frac{1}{\sigma L_{0}^{2}}\|p\|^{2}+\frac{1}{\sigma}\|\nabla p\|^{2} & \text { when } \nu=0 .
\end{array}
$$

2. Stability in $\|\cdot\|$ for the solution of problem (2.5) will be obtained provided the data are regular enough, that is, $f \in V_{\mathcal{L}}^{\prime}$ and $g \in L^{2}(\Omega)^{\prime}=L^{2}(\Omega)$. If the data are less regular, stability for $[\boldsymbol{u}, p]$ can be proved in norms weaker than $\|\cdot\| \cdot$, and therefore $[\boldsymbol{u}, p]$ will belong to larger functional spaces than $V \times Q$. In particular, for $\sigma=0$, we may take $f \in H^{-1}(\Omega)^{d}$ but for $\nu=0$, if $\boldsymbol{f} \in H_{0}(\operatorname{div}, \Omega)^{\prime}$, control on $\frac{1}{\sigma}\|\nabla p\|^{2}$ is lost, whereas if $g \in H^{-1}(\Omega)$ (mixed formulation of the Poisson problem) control on $\sigma L_{0}^{2}\|\nabla \cdot \boldsymbol{u}\|^{2}$ is lost $\left(\boldsymbol{u} \in L^{2}(\Omega)^{d}\right.$ only, we do not have $\left.\boldsymbol{u} \in H_{0}(\operatorname{div}, \Omega)\right)$. These facts are easily deduced from the proof of Theorem 2.1.

Copyright $@$ ㅇ by SIAM. Unauthorized reproduction of this article is prohibited. 


\section{Analysis of stabilized finite element method for the generalized Stokes-Darcy problem.}

3.1. Preliminaries. Let us introduce some notation. The finite element partition will be denoted by $\mathcal{P}_{h}=\{K\}$, and summation over all the elements will be indicated as $\sum_{K}$. The collection of all interior edges (faces, for $d=3$ ) will be denoted by $\mathcal{E}_{h}=\{E\}$, and as for the elements, summation over all these edges will be indicated as $\sum_{E}$. Consistently with the notation introduced previously, the symbol $\left\langle f_{1}, f_{2}\right\rangle_{D}$ will be used to denote the integral of the product of functions $f_{1}$ and $f_{2}$ over $D$, with $D=K$ (an element), $D=\partial K$ (an element boundary), or $D=E$ (an edge). Likewise, $\left\|f_{1}\right\|_{D}^{2}:=\left\langle f_{1}, f_{1}\right\rangle_{D}$. Suppose now that elements $K_{1}$ and $K_{2}$ share an edge $E$, and let $\boldsymbol{n}_{1}$ and $\boldsymbol{n}_{2}$ be the normals to $E$ exterior to $K_{1}$ and $K_{2}$, respectively. For a scalar function $f$, possibly discontinuous across $E$, we define its jump as $\llbracket n f \rrbracket_{E}:=\left.n_{1} f\right|_{\partial K_{1} \cap E}+\left.n_{2} f\right|_{\partial K_{2} \cap E}$, and for a vector or tensor $v$, $\llbracket n \cdot v \rrbracket_{E}:=\left.\boldsymbol{n}_{1} \cdot \boldsymbol{v}\right|_{\partial K_{1} \cap E}+\left.n_{2} \cdot v\right|_{\partial K_{2} \cap E}$.

We will consider, for the sake of conciseness, quasi-uniform finite element partitions (for the analysis of the stabilized formulation in the more general nondegenerate case, see [13]). Therefore, we assume that there is a constant $C_{\text {inv }}$, independent of the mesh size $h$ (the maximum of all the element diameters), such that

\section{(3.1) $\quad\left\|\nabla v_{h}\right\|_{K} \leq C_{\mathrm{inv}} h^{-1}\left\|v_{h}\right\|_{K}, \quad\left\|\Delta v_{h}\right\|_{K} \leq C_{\mathrm{inv}} h^{-1}\left\|\nabla v_{h}\right\|_{K}$}

\section{$\mathbf{S}$
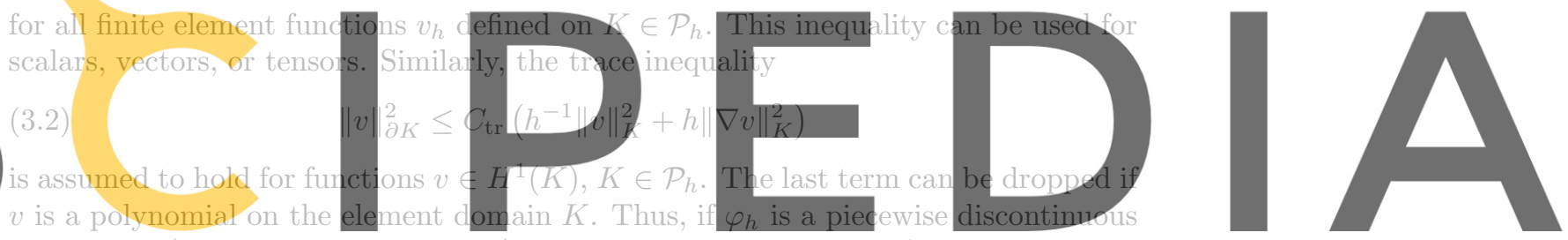 \\ polynomial (the pressure, in our case) and $\psi_{h}$ a continuous one, it follows that

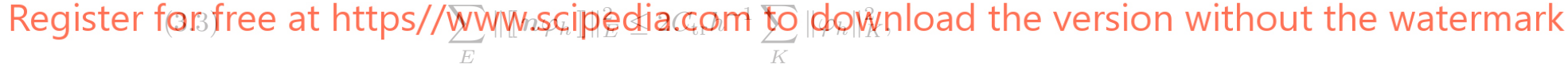

$$
\sum_{E}\left\|\psi_{h}\right\|_{E}^{2} \leq \frac{1}{2} C_{\mathrm{tr}} h^{-1} \sum_{K}\left\|\psi_{h}\right\|_{K}^{2} .
$$

Given a function $v$, let $\tilde{v}_{h}$ be an interpolant of $v$ in the finite element space. Using the trace inequality (3.2) we have that

$$
\begin{aligned}
\sum_{E}\left\|\llbracket \boldsymbol{n}\left(v-\tilde{v}_{h}\right) \rrbracket\right\|_{E}^{2} & \leq 2 \sum_{K}\left\|v-\tilde{v}_{h}\right\|_{\partial K}^{2} \\
& \leq 2 C_{\operatorname{tr}} \sum_{K}\left(h^{-1}\left\|v-\tilde{v}_{h}\right\|_{K}^{2}+h\left\|\nabla v-\nabla \tilde{v}_{h}\right\|_{K}^{2}\right),
\end{aligned}
$$

so that we obtain the two expressions we will use:

$$
\sum_{E}\left\|\llbracket \boldsymbol{n}\left(v-\tilde{v}_{h}\right) \rrbracket\right\|_{E}^{2} \lesssim\left(h^{-1} \varepsilon_{0}^{2}(v)+h \varepsilon_{1}^{2}(v)\right) \lesssim h^{2 j-1}|v|_{H^{j}(\Omega)}^{2}, j=1,2,
$$

where $\varepsilon_{i}(v)=\left|v-\tilde{v}_{h}\right|_{H^{i}(\Omega)},|\cdot|_{H^{i}(\Omega)}$ being the seminorm in $H^{i}(\Omega)$. The same estimate holds for a continuous interpolation:

$$
\sum_{E}\left\|\left(v-\tilde{v}_{h}\right)\right\|_{E}^{2} \lesssim\left(h^{-1} \varepsilon_{0}^{2}(v)+h \varepsilon_{1}^{2}(v)\right) .
$$

Copyright $@$ by SIAM. Unauthorized reproduction of this article is prohibited. 
3.2. Stabilized finite element methods. In this subsection we describe the two stabilized finite element methods we shall consider. As it has been mentioned, both can be cast in the variational multiscale framework [20, 21]. However, we will not present the motivation, which can be found in [2].

Let $V_{h}$ and $Q_{h}$ be the finite element spaces to approximate the velocity and the pressure, respectively. The two methods to be analyzed can be written as follows: find $\left[\boldsymbol{u}_{h}, p_{h}\right] \in V_{h} \times Q_{h}$ such that

$$
B_{s}\left(\left[\boldsymbol{u}_{h}, p_{h}\right],\left[\boldsymbol{v}_{h}, q_{h}\right]\right)=L_{s}\left(\left[\boldsymbol{v}_{h}, q_{h}\right]\right)
$$

$\forall\left[\boldsymbol{v}_{h}, q_{h}\right] \in V_{h} \times Q_{h}$. The bilinear form $B_{s}$ and the linear form $L_{s}$ are modifications of $B$ and $L$ (defined in (2.6) and (2.7), respectively) that depend on the stabilized formulation being used.

\subsubsection{ASGS method. In this case, the forms $B_{s}$ and $L_{s}$ are given by}

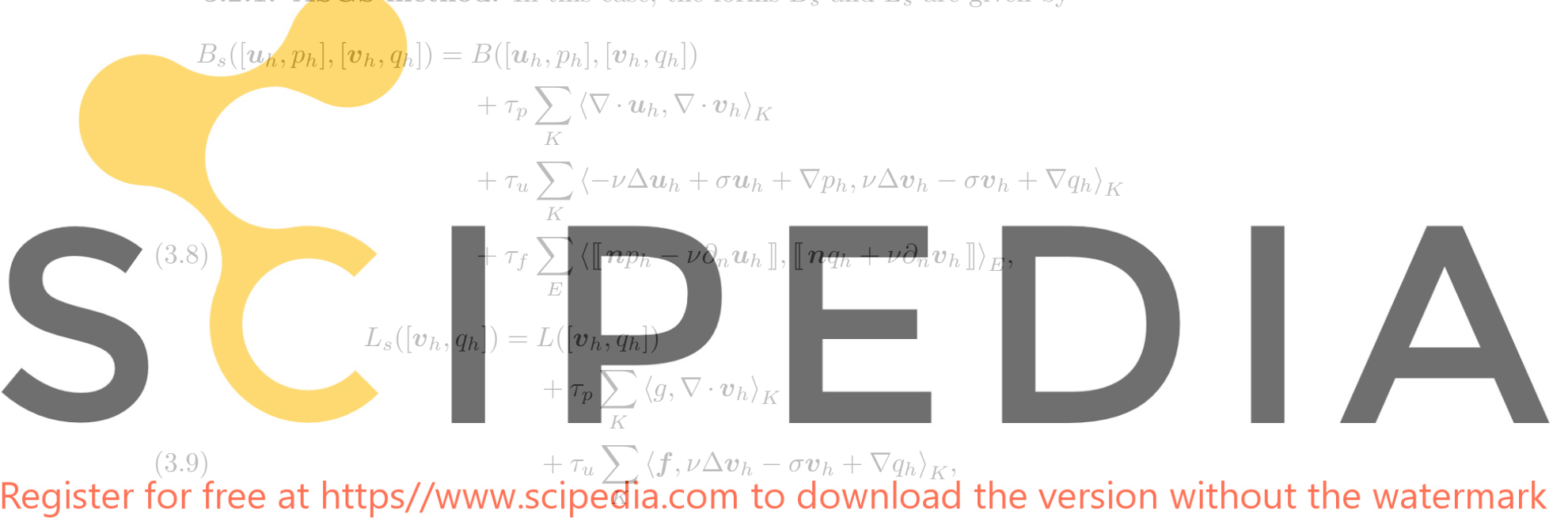

where $\tau_{p}, \tau_{u}$, and $\tau_{f}$ are the so-called stabitization parameters that we compute as

$$
\begin{aligned}
\tau_{p} & =c_{1} \nu+c_{2}^{p} \sigma \ell_{p}^{2}, \\
\tau_{u} & =\left(c_{1} \nu+c_{2}^{u} \sigma \ell_{u}^{2}\right)^{-1} h^{2}, \\
\tau_{f} & =\left(c_{1} \nu+c_{2}^{u} \sigma \ell_{u}^{2}\right)^{-1} h,
\end{aligned}
$$

with $c_{1}, c_{2}^{p}$, and $c_{2}^{u}$ algorithmic constants. In these expressions we have introduced the length scales $\ell_{u}$ and $\ell_{p}$, which can be taken either as $L_{0}, h$ or $\left(L_{0} h\right)^{1 / 2}$; these two length scales cannot be chosen independently because they have to satisfy some conditions that are required in the numerical analysis. Even though it is not our purpose here to motivate the introduction of these length scales, let us briefly mention which is their origin (see [2] for a more detailed description in the Darcy case). In order to define a norm in the space of forcing terms (or, equivalently, in the space of finite element residuals) it is necessary to introduce scaling coefficients $\mu_{u}$ and $\mu_{p}$ such that $\mu_{u}|\boldsymbol{f}|^{2}+\mu_{p}|g|^{2}$ is dimensionally consistent. This scaling is necessary in order to obtain approximations for the subscales on which the method is based, in the spirit of $[20,12]$. Using the approximate Fourier analysis proposed in [12], the stabilization parameters (3.10)-(3.11) are found, now depending on $\mu_{u}$ and $\mu_{p}$. In turn, these scaling coefficients depend on a length scale of the problem that may be taken as $L_{0}$ 
or $h$. The different choices lead to the different possibilities we will consider for $\ell_{u}$ and $\ell_{p}$.

Concerning the design of (3.12) and, in fact, of the terms involving the jumps across interelement edges in (3.8), their motivation can be found in [15]. Note, in particular, the change in the sign of $-\nu \partial_{n} \boldsymbol{u}_{h}$ and $\nu \partial_{n} \boldsymbol{v}_{h}$.

Let us also mention that we have considered the stabilization parameters constant for the sake of simplicity. If the mesh is not quasi-uniform, they should be computed elementwise (see [13]). In the case of variable viscosity or permeability, the approach proposed could be extended evaluating these parameters pointwise, inside the element integrals.

3.2.2. OSS method. Let us denote by $P$ the projection onto the finite element space of either velocities or pressures. Whether it is one case or the other will be determined by whether $P$ is applied to vectors (first case) or to scalars (second case).

The bilinear form $B_{s}$ and the linear form $L_{s}$ in the OSS method are given by

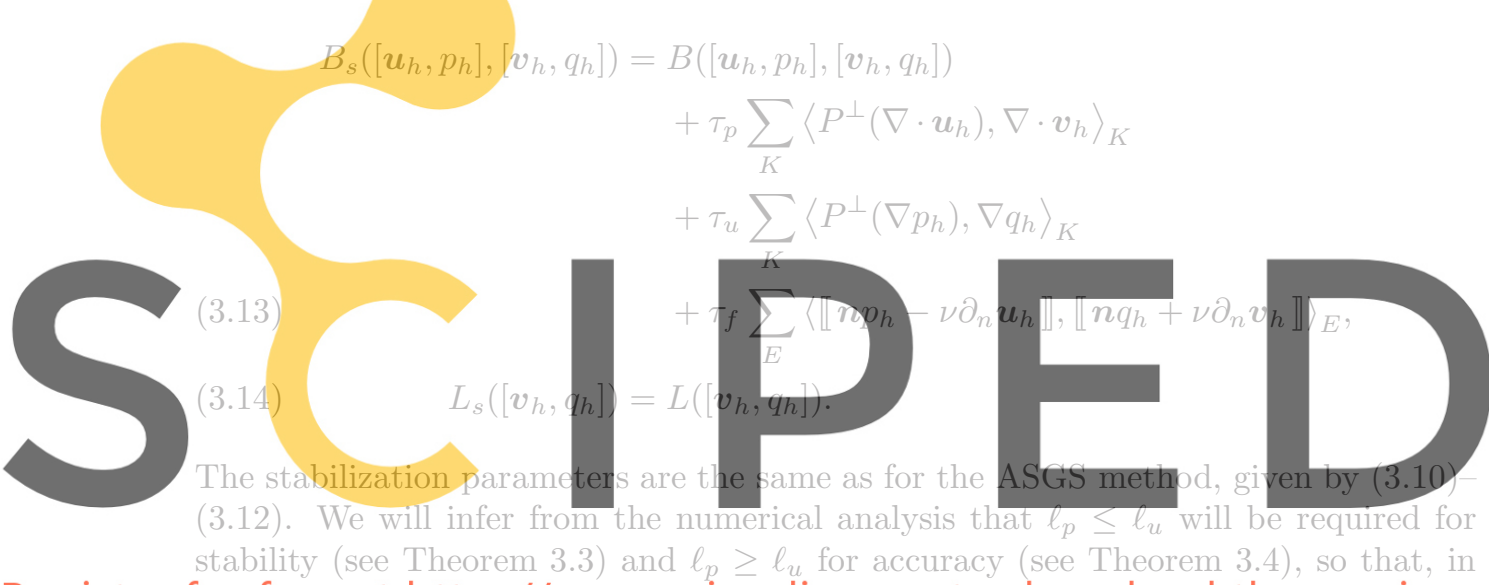

\section{Register fon freerith hattpos dqwWw.seipedia.com to download the version without the watermark}

\subsubsection{Working norm and error function. Let us define the mesh dependent} norm

$$
\begin{aligned}
\left\|\left[\boldsymbol{v}_{h}, q_{h}\right]\right\|_{h}^{2}= & \left\|\boldsymbol{v}_{h}\right\|_{\mathcal{L}}^{2}+\sigma \ell_{p}^{2}\left\|\nabla \cdot \boldsymbol{v}_{h}\right\|^{2}+\frac{1}{\nu+\sigma L_{0}^{2}}\left\|q_{h}\right\|^{2} \\
& +\frac{h^{2}}{\nu+\sigma \ell_{u}^{2}} \sum_{K}\left\|\nabla q_{h}\right\|_{K}^{2}+\frac{h}{\nu+\sigma \ell_{u}^{2}} \sum_{E}\left\|\llbracket \boldsymbol{n} q_{h} \rrbracket\right\|_{E}^{2},
\end{aligned}
$$

where $\ell_{p}$ and $\ell_{u}$ are the length scales introduced in the stabilization parameters (3.10)(3.12). This is precisely the norm in which the numerical analysis will be performed, that is to say, stability and convergence will be proved in this norm, both for the ASGS and the OSS methods.

We define

$$
E(h)^{2}=\left(\nu+\sigma \ell_{p}^{2}\right)\left(h^{-2} \varepsilon_{0}^{2}(\boldsymbol{u})+\varepsilon_{1}^{2}(\boldsymbol{u})\right)+\sigma \varepsilon_{0}^{2}(\boldsymbol{u})+\frac{h^{2}}{\nu+\sigma \ell_{u}^{2}}\left(h^{-2} \varepsilon_{0}^{2}(p)+\varepsilon_{1}^{2}(p)\right) .
$$

It will be proved that this is precisely the error function of the method in the previous norm (recall that $\varepsilon_{i}(v)$ is the interpolation error of function $v$ in the norm of $H^{i}(\Omega)$ ). 
3.2.4. Simplified methods for continuous pressures in the Darcy problem. Both the ASGS and the OSS methods can be simplified in the case of continuous pressures and Darcy's problem in the case $\tau_{p}=0$. Assuming $\boldsymbol{f} \in L^{2}(\Omega)^{d}$ and $g \in L^{2}(\Omega)$, it is immediately checked that these methods reduce to the following:

$A S G S$ method. In this case

$$
\begin{gathered}
\sigma\left(\boldsymbol{u}_{h}, \boldsymbol{v}_{u}\right)+\left(\nabla p_{h}, \boldsymbol{v}_{h}\right)-\left(\nabla q_{h}, \boldsymbol{u}_{h}\right)+\frac{1}{\sigma c_{2}^{u}}\left(\sigma \boldsymbol{u}_{h}+\nabla p_{h},-\sigma \boldsymbol{v}_{h}+\nabla q_{h}\right) \\
=\left(\boldsymbol{v}_{h}, \boldsymbol{f}\right)+\frac{1}{\sigma c_{2}^{u}}\left(-\sigma \boldsymbol{v}_{h}+\nabla q_{h}, \boldsymbol{f}\right)+\left(q_{h}, g\right),
\end{gathered}
$$

from where

$$
\begin{aligned}
& \left(1-\frac{1}{c_{2}^{u}}\right) B\left(\left[\boldsymbol{u}_{h}, p_{h}\right],\left[\boldsymbol{v}_{h}, q_{h}\right]\right)+\frac{1}{\sigma c_{2}^{u}}\left(\nabla q_{h}, \nabla p_{h}\right) \\
& =\left(1-\frac{1}{c_{2}^{u}}\right) L\left(\left[v_{h}, q_{h}\right]\right)+\frac{1}{\sigma c_{2}^{u}}\left(\nabla q_{h}, f\right)+\frac{1}{c_{2}^{u}}\left(q_{h}, g\right),
\end{aligned}
$$

which can be understood as the a combination

$$
\left.(1-\alpha) \text { Galerkin equation }+\alpha \text { (weak form of }-\frac{1}{\sigma} \Delta p_{h}=-\frac{1}{\sigma} \nabla \cdot f+g\right),
$$

with $\alpha=1 / c_{2}^{u}$. We obtain precisely the method called Galerkin stabilization in [4] (with a different definition of the corresponds, in fact, to Note that the method is OSS method. In this $\sigma\left(u_{h}, v_{u}\right)+\left(\nabla p_{h}, v_{h}\right)$
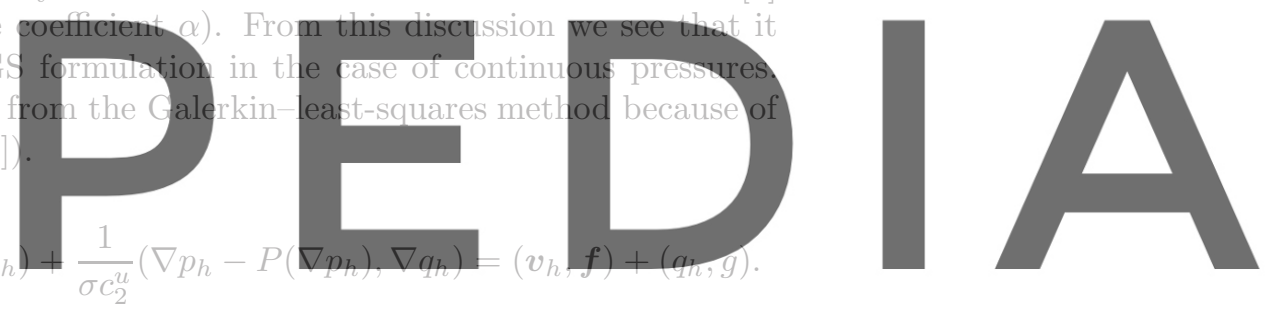

Register for free at hittps/\% wWw.scipedia.com to download the version without the watermark

from where

$$
\begin{array}{r}
\sigma\left(\boldsymbol{u}_{h}, \boldsymbol{v}_{h}\right)+\left(\nabla p_{h}, \boldsymbol{v}_{h}\right)-\left(1-\frac{1}{c_{2}^{u}}\right)\left(\nabla q_{h}, \boldsymbol{u}_{h}\right)+\frac{1}{\sigma c_{2}^{u}}\left(\nabla q_{h}, \nabla p_{h}\right) \\
=\left(\boldsymbol{v}_{h}, \boldsymbol{f}\right)+\left(1-\frac{1}{c_{2}^{u}}\right)\left(q_{h}, g\right)+\frac{1}{\sigma c_{2}^{u}}\left(\nabla q_{h}, P(\boldsymbol{f})\right)+\frac{1}{c_{2}^{u}}\left(q_{h}, g\right),
\end{array}
$$

which can be understood as

first Galerkin equation $+(1-\alpha)$ second Galerkin equation

$$
+\alpha\left(\text { weak form of }-\frac{1}{\sigma} \Delta p_{h}=-\frac{1}{\sigma} \nabla \cdot P(\boldsymbol{f})+g\right),
$$

with $\alpha=1 / c_{2}^{u}$ and where the first Galerkin equation refers to that obtained with $q_{h}=0$ and the second to the one obtained with $\boldsymbol{v}_{h}=\mathbf{0}$. If we redefine $q_{h}$ appropriately, we see that the method coincides with (3.17) except for the projection $P$ applied to $f$, which is usually constant and belongs to the finite element space for Darcy flow. Apart from this detail, when $\alpha=1$, the OSS method is equivalent to the standard Galerkin approximation of the second order elliptic problem (only in terms of $p$ ) using $Q_{h}$ and a postprocess of fluxes using an $L^{2}$-projection of $\nabla p_{h}$ onto $V_{h}$; for the ASGS approximation, this choice leads to a singular problem. 
3.3. Analysis of the ASGS formulation. In this section we will state and prove two main theorems, both for the ASGS and the OSS methods, one establishing stability in the form of inf-sup condition and the other given convergence in the norm (3.15). We start in this section with the ASGS method.

THEOREM 3.1 (stability of the ASGS formulation). Suppose that the constants $c_{1}$ and $c_{2}^{u}$ in (3.10)-(3.11) are large enough (in particular, $c_{1}>2\left(C_{\mathrm{inv}}^{2}+C_{\mathrm{tr}}\right.$ ) and $c_{2}^{u}>2$, with $C_{\mathrm{inv}}$ and $C_{\mathrm{tr}}$ introduced in (3.1) and (3.2), respectively). Then, there exists a constant $C$ such that

$$
\forall\left[\boldsymbol{u}_{h}, p_{h}\right] \text { there exists }\left[\boldsymbol{v}_{h}, q_{h}\right] \mid B_{s}\left(\left[\boldsymbol{u}_{h}, p_{h}\right],\left[\boldsymbol{v}_{h}, q_{h}\right]\right) \geq C\left\|\left[\boldsymbol{u}_{h}, p_{h}\right]\right\|\left\|_{h}\right\|\left[\boldsymbol{v}_{h}, q_{h}\right]\|\|_{h},
$$
where $B_{s}$ is defined in (3.8).

Proof. Let us start taking the test function equal to the unknown, using the inverse estimate (3.1), the trace inequality (3.3), and Schwartz and Young's inequalities:

$$
\begin{aligned}
& B_{s}\left(\left[\boldsymbol{u}_{h}, p_{h}\right],\left[\boldsymbol{u}_{h}, p_{h}\right]\right) \\
& =\nu\left\|\nabla \boldsymbol{u}_{h}\right\|^{2}+\sigma\left\|\boldsymbol{u}_{h}\right\|^{2}+\tau_{u} \sum_{K}\left(\left\|\nabla p_{h}\right\|_{K}^{2}-\left\|-\nu \Delta \boldsymbol{u}_{h}+\sigma \boldsymbol{u}_{h}\right\|_{K}^{2}\right) \\
& \quad+\tau_{p}\left\|\nabla \cdot \boldsymbol{u}_{h}\right\|^{2}+\tau_{f} \sum_{E}\left\|\llbracket \boldsymbol{n} p_{h} \rrbracket\right\|_{E}^{2}-\tau_{f} \nu^{2} \sum_{E}\left\|\llbracket \partial_{h} \boldsymbol{u}_{h} \rrbracket\right\|_{E}^{2} \\
& \geq \\
& \quad \nu\left(1-2 \tau_{u} \nu \frac{C_{\mathrm{inv}}^{2}}{h^{2}}-2 \tau_{f} \nu \frac{C_{\mathrm{tr}}}{h}\right)\left\|\nabla \boldsymbol{u}_{h}\right\|^{2}+\sigma\left(1-2 \tau_{u} \sigma\right)\left\|\boldsymbol{u}_{h}\right\|^{2}+\tau_{p}\left\|\nabla \cdot \boldsymbol{u}_{h}\right\|^{2} \\
& \quad+\tau_{u} \sum_{K}\left\|\nabla p_{h}\right\|_{K}^{2}+\tau_{f} \sum_{E}\left\|\llbracket \boldsymbol{n} p_{h} \rrbracket\right\|_{E}^{2} .
\end{aligned}
$$

The coefficients within parenthesis are positive for any choice of physical parameters, provided (sufficient conditions)

$$
c_{1}>2\left(C_{\mathrm{inv}}^{2}+C_{\mathrm{tr}}\right), \quad c_{2}^{u}>\frac{2 h^{2}}{\ell_{u}^{2}} .
$$

The second condition reduces to $c_{2}^{u}>2$ for $\ell_{u}=h$. For the other choices of $\ell_{u}$, the second condition always holds for a small enough $h$. Note that we have used that $\tau_{f}=\tau_{u} / h$.

The only term missing in the right-hand side of (3.19) from $\|\cdot\|_{h}^{2}$ is the $L^{2}$-norm of the pressure. In order to introduce it, let us note that from the inf-sup condition for the continuous problem, it turns out that for all $p_{h} \in Q_{h}$, there exists $\boldsymbol{v}_{p} \in V$ such that

$$
-\left(p_{h}, \nabla \cdot \boldsymbol{v}_{p}\right) \gtrsim\left\|p_{h}\right\| \frac{1}{\sqrt{\nu+\sigma L_{0}^{2}}}\left(\sqrt{\nu+\sigma \ell_{u}^{2}}\left\|\nabla \boldsymbol{v}_{p}\right\|+\sqrt{\sigma}\left\|\boldsymbol{v}_{p}\right\|+\sqrt{\sigma} L_{0}\left\|\nabla \cdot \boldsymbol{v}_{p}\right\|\right) .
$$

We may choose $\boldsymbol{v}_{p}$ such that

$$
\sqrt{\nu+\sigma \ell_{u}^{2}}\left\|\nabla \boldsymbol{v}_{p}\right\|+\sqrt{\sigma}\left\|\boldsymbol{v}_{p}\right\|+\sqrt{\sigma} L_{0}\left\|\nabla \cdot \boldsymbol{v}_{p}\right\|=\frac{1}{\sqrt{\nu+\sigma L_{0}^{2}}}\left\|p_{h}\right\| .
$$

Let $\tilde{\boldsymbol{v}}_{p, h}$ be a $H^{1}$-continuous finite element interpolant of $\boldsymbol{v}_{p}$, e.g., defined by the Scott-Zhang or the Clément operator (see [19]). Because of the continuity of this interpolant,

$$
\sqrt{\nu+\sigma \ell_{u}^{2}}\left\|\nabla \tilde{\boldsymbol{v}}_{p, h}\right\|+\sqrt{\sigma}\left\|\tilde{\boldsymbol{v}}_{p, h}\right\|+\sqrt{\sigma} L_{0}\left\|\nabla \cdot \tilde{\boldsymbol{v}}_{p, h}\right\| \lesssim \frac{1}{\sqrt{\nu+\sigma L_{0}^{2}}}\left\|p_{h}\right\| .
$$

Copyright (c) by SIAM. Unauthorized reproduction of this article is prohibited. 
For $\left[\boldsymbol{v}_{2}, q_{2}\right]=\left[\tilde{\boldsymbol{v}}_{p, h}, 0\right]$, we have

$$
\begin{aligned}
& B_{s}\left(\left[\boldsymbol{u}_{h}, p_{h}\right],\left[\boldsymbol{v}_{2}, q_{2}\right]\right) \\
& \geq \\
& \quad-\nu\left\|\nabla \boldsymbol{u}_{h}\right\|\left\|\nabla \tilde{\boldsymbol{v}}_{p, h}\right\|-\sigma\left\|\boldsymbol{u}_{h}\right\|\left\|\tilde{\boldsymbol{v}}_{p, h}\right\|-\tau_{p}\left\|\nabla \cdot \boldsymbol{u}_{h}\right\|\left\|\nabla \cdot \tilde{\boldsymbol{v}}_{p, h}\right\| \\
& \quad+\tau_{u} \sum_{K}\left\langle-\nu \Delta \boldsymbol{u}_{h}+\sigma \boldsymbol{u}_{h}+\nabla p_{h}, \nu \Delta \tilde{\boldsymbol{v}}_{p, h}-\sigma \tilde{\boldsymbol{v}}_{p, h}\right\rangle_{K} \\
& \quad+\tau_{f} \sum_{E}\left\langle\llbracket \boldsymbol{n} p_{h}-\nu \partial_{h} \boldsymbol{u}_{h} \rrbracket, \nu \llbracket \partial_{h} \tilde{\boldsymbol{v}}_{p, h} \rrbracket\right\rangle_{E} \\
& \quad-\left(p_{h}, \nabla \cdot \boldsymbol{v}_{p}\right)-\left(p_{h}, \nabla \cdot\left(\tilde{\boldsymbol{v}}_{p, h}-\boldsymbol{v}_{p}\right)\right) .
\end{aligned}
$$

The term that provides pressure stability is

$$
-\left(p_{h}, \nabla \cdot \boldsymbol{v}_{p}\right) \gtrsim \frac{1}{\nu+\sigma L_{0}^{2}}\left\|p_{h}\right\|^{2},
$$

by virtue of the choice of the norm of $\boldsymbol{v}_{p}$. Let us bound the different terms appearing in (3.20). Using Young's inequality we will have that

$$
\begin{aligned}
- & \nu\left\|\nabla \boldsymbol{u}_{h}\right\|\left\|\nabla \tilde{\boldsymbol{v}}_{p, h}\right\|-\sigma\left\|\boldsymbol{u}_{h}\right\|\left\|\tilde{\boldsymbol{v}}_{p, h}\right\|-\tau_{p}\left\|\nabla \cdot \boldsymbol{u}_{h}\right\|\left\|\nabla \cdot \tilde{\boldsymbol{v}}_{p, h}\right\| \\
& \gtrsim-\beta_{1}\left(\nu\left\|\nabla \boldsymbol{u}_{h}\right\|^{2}+\sigma\left\|\boldsymbol{u}_{h}\right\|^{2}+\tau_{p}\left\|\nabla \cdot \boldsymbol{u}_{h}\right\|^{2}\right) \\
& -\frac{1}{\beta_{1}}\left(\nu\left\|\nabla \tilde{\boldsymbol{v}}_{p, h}\right\|^{2}+\sigma\left\|\tilde{\boldsymbol{v}}_{p, h}\right\|^{2}+\tau_{p}\left\|\nabla \cdot \tilde{\boldsymbol{v}}_{p, h}\right\|^{2}\right) \\
& \gtrsim-\beta_{1}\left(\nu\left\|\nabla \boldsymbol{u}_{h}\right\|^{2}+\sigma\left\|\boldsymbol{u}_{h}\right\|^{2}+\tau_{p}\left\|\nabla \cdot \boldsymbol{u}_{h}\right\|^{2}\right)-\frac{1}{\beta_{1}} \frac{1}{\nu+\sigma L_{0}^{2}}\left\|p_{h}\right\|^{2}
\end{aligned}
$$

for any constant $\beta_{1}>0$. In the following we will denote by $\beta_{i}, i=1,2,3,4$, constants appearing from the application of Young's inequality that, at the end, will be chosen to be sufficiently large.

The next term to bound in (3.20) is

$$
\begin{aligned}
\tau_{u} \sum_{K}\left\langle-\nu \Delta \boldsymbol{u}_{h}+\sigma \boldsymbol{u}_{h}+\nabla p_{h}, \nu \Delta \tilde{\boldsymbol{v}}_{p, h}-\sigma \tilde{\boldsymbol{v}}_{p, h}\right\rangle_{K} \\
\gtrsim-\beta_{2} \tau_{u} \sum_{K}\left(\nu^{2} \frac{C_{\mathrm{inv}}^{2}}{h^{2}}\left\|\nabla \boldsymbol{u}_{h}\right\|_{K}^{2}+\sigma^{2}\left\|\boldsymbol{u}_{h}\right\|_{K}^{2}+\left\|\nabla p_{h}\right\|_{K}^{2}\right) \\
\quad-\frac{1}{\beta_{2}} \tau_{u} \sum_{K}\left(\nu^{2} \frac{C_{\mathrm{inv}}^{2}}{h^{2}}\left\|\nabla \tilde{\boldsymbol{v}}_{p, h}\right\|_{K}^{2}+\sigma^{2}\left\|\tilde{\boldsymbol{v}}_{p, h}\right\|_{K}^{2}\right) \\
\gtrsim-\beta_{2}\left(\nu\left\|\nabla \boldsymbol{u}_{h}\right\|^{2}+\sigma\left\|\boldsymbol{u}_{h}\right\|^{2}+\tau_{u} \sum_{K}\left\|\nabla p_{h}\right\|_{K}^{2}\right)-\frac{1}{\beta_{2}} \frac{1}{\nu+\sigma L_{0}^{2}}\left\|p_{h}\right\|^{2},
\end{aligned}
$$

where we have used the fact that $c_{1} \tau_{u} \nu<h^{2}$ and $c_{2}^{u} \tau_{u} \sigma<1$. We can also bound

$$
\begin{aligned}
\tau_{f} & \sum_{E}\left\langle\llbracket \boldsymbol{n} p_{h}-\nu \partial_{n} \boldsymbol{u}_{h} \rrbracket, \nu \llbracket \partial_{n} \tilde{\boldsymbol{v}}_{p, h} \rrbracket\right\rangle_{E} \\
& \gtrsim-\beta_{3} \tau_{f} \sum_{E}\left\|\llbracket \boldsymbol{n} p_{h} \rrbracket\right\|_{E}^{2}-\beta_{3} \tau_{f} \nu^{2} \sum_{E}\left\|\llbracket \partial_{n} \boldsymbol{u}_{h} \rrbracket\right\|_{E}^{2}-\frac{1}{\beta_{3}} \tau_{f} \nu^{2} \sum_{E}\left\|\llbracket \partial_{n} \tilde{\boldsymbol{v}}_{p, h} \rrbracket\right\|_{E}^{2} \\
& \geq-\beta_{3} \tau_{f} \sum_{E}\left\|\llbracket \boldsymbol{n} p_{h} \rrbracket\right\|_{E}^{2}-\beta_{3} \nu\left\|\nabla \boldsymbol{u}_{h}\right\|^{2}-\frac{1}{\beta_{3}} \frac{1}{\nu+\sigma L_{0}^{2}}\left\|p_{h}\right\|^{2}
\end{aligned}
$$

Copyright $(\odot$ by SIAM. Unauthorized reproduction of this article is prohibited. 
where we have made use of (3.3). Finally,

$$
\begin{gathered}
-\left(p_{h}, \nabla \cdot\left(\tilde{\boldsymbol{v}}_{p, h}-\boldsymbol{v}_{p}\right)\right)=\sum_{K}\left\langle\nabla p_{h}, \tilde{\boldsymbol{v}}_{p, h}-\boldsymbol{v}_{p}\right\rangle_{K}-\sum_{K}\left\langle\boldsymbol{n} p_{h}, \tilde{\boldsymbol{v}}_{p, h}-\boldsymbol{v}_{p}\right\rangle_{\partial K} \\
\geq-\sum_{K}\left\|\nabla p_{h}\right\|_{K}\left\|\tilde{\boldsymbol{v}}_{p, h}-\boldsymbol{v}_{p}\right\|_{K}-\sum_{E}\left\|\llbracket \boldsymbol{n} p_{h} \rrbracket\right\|_{E}\left\|\tilde{\boldsymbol{v}}_{p, h}-\boldsymbol{v}_{p}\right\|_{E} \\
\gtrsim-\frac{\beta_{4} h^{2}}{\nu+\sigma \ell_{u}^{2}} \sum_{K}\left\|\nabla p_{h}\right\|_{K}^{2}-\frac{\beta_{4} h}{\nu+\sigma \ell_{u}^{2}} \sum_{E}\left\|\llbracket \boldsymbol{n} p_{h} \rrbracket\right\|_{E}^{2}-\frac{1}{\beta_{4}}\left(\nu+\sigma \ell_{u}^{2}\right)\left\|\nabla \boldsymbol{v}_{p}\right\|^{2} \\
\gtrsim-\frac{\beta_{4} h^{2}}{\nu+\sigma \ell_{u}^{2}} \sum_{K}\left\|\nabla p_{h}\right\|_{K}^{2}-\frac{\beta_{4} h}{\nu+\sigma \ell_{u}^{2}} \sum_{E}\left\|\llbracket \boldsymbol{n} p_{h} \rrbracket\right\|_{E}^{2}-\frac{1}{\beta_{4}} \frac{1}{\nu+\sigma L_{0}^{2}}\left\|p_{h}\right\|^{2},
\end{gathered}
$$

where we have used Young's inequality, the first inverse estimate in (3.1), and the trace inequality in (3.2). The theorem follows taking the constants $\beta_{i}, i=1,2,3,4$, sufficiently large, combining (3.21)-(3.25) in (3.20) and the result with (3.19).

Remark 3.1. Let us compare the working norms of the continuous and the discrete problems, for simplicity in the case of continuous pressure interpolations:

$$
\begin{gathered}
\|[\boldsymbol{v}, q]\|^{2}=\|\boldsymbol{v}\|_{\mathcal{L}}^{2}+\sigma L_{0}^{2}\|\nabla \cdot \boldsymbol{v}\|^{2}+\frac{1}{\nu+\sigma L_{0}^{2}}\|q\|^{2}+\|\nabla q\|_{\mathcal{L}^{\prime}}^{2}, \\
\left\|\left[\boldsymbol{v}_{h}, q_{h}\right]\right\|_{h}^{2}=\left\|\boldsymbol{v}_{h}\right\|_{\mathcal{L}}^{2}+\sigma \ell_{p}^{2}\left\|\nabla \cdot \boldsymbol{v}_{h}\right\|^{2}+\frac{1}{\nu+\sigma L_{0}^{2}}\left\|q_{h}\right\|^{2}+\frac{h^{2}}{\nu+\sigma \ell_{u}^{2}}\left\|\nabla q_{h}\right\|^{2} .
\end{gathered}
$$

We have the following options:

- $\ell_{p}=L_{0}$ and $\ell_{u}=h$. The discrete norm would reproduce the continuous norm, provided the discrete counterpart of $\|\nabla q\|_{\mathcal{L}^{\prime}}^{2}$ is considered to be $\left(\nu / h^{2}+\right.$ $\sigma)^{-1}\left\|\nabla q_{h}\right\|^{2}$. This is obviously the case when $\nu=0$. When $\nu>0$, the $H^{-1}(\Omega)$-norm of $\nabla q$ has to be replaced by $h^{2}\left\|\nabla q_{h}\right\|^{2}$.

- $\ell_{p}=L_{0}$ and $\ell_{u}=L_{0}$. We loose pressure stability in $H^{1}(\Omega)$ when $\nu=0$, but the rest of terms are optimal.

- $\ell_{p}=h$ and $\ell_{u}=L_{0}$. We loose pressure stability in $H^{1}(\Omega)$ and velocity stability in $H(\operatorname{div}, \Omega)$ when $\nu=0$. We will see that this case is not allowed by the convergence analysis.

- $\ell_{p}=h$ and $\ell_{u}=h$. We loose velocity stability in $H(\operatorname{div}, \Omega)$ when $\nu=0$, but the rest of terms are optimal.

Even if it is difficult to motivate the choice from the stability analysis, we will see in section 4 that from the accuracy point of view the optimal choice is $\ell_{p}=\ell_{u}=\left(L_{0} h\right)^{1 / 2}$ when equal velocity-pressure interpolation is used.

THEOREM 3.2 (convergence of the ASGS formulation). Let $[\boldsymbol{u}, p]$ be the solution of the continuous problem (2.5) and $\left[\boldsymbol{u}_{h}, p_{h}\right]$ the solution of (3.7) with $B_{s}$ and $L_{s}$ given in (3.8) and (3.9), respectively. Suppose that $\ell_{p} \geq \ell_{u}$ and the assumptions of Theorem 3.1 hold. Then

$$
\left\|\left[\boldsymbol{u}-\boldsymbol{u}_{h}, p-p_{h}\right]\right\|_{h} \lesssim E(h)
$$

where the error function $E(h)$ is given in (3.16).

Copyright $@$ by SIAM. Unauthorized reproduction of this article is prohibited. 
Proof. Let $\left[\tilde{\boldsymbol{u}}_{h}, \tilde{p}_{h}\right]$ be the interpolant of $[\boldsymbol{u}, p]$. By definition of $E(h)$ and $\|\cdot\|_{h}$ and the fact that $\ell_{u} \leq L_{0}$,

$$
\left\|\left[\boldsymbol{u}-\tilde{\boldsymbol{u}}_{h}, p-\tilde{p}_{h}\right]\right\|_{h} \lesssim E(h),
$$

where we have used (3.5). This is, in fact, the inequality that determines the form of the error function $E(h)$.

On the other hand, there exists $\left[\boldsymbol{v}_{h}, q_{h}\right]$ such that

$$
\begin{aligned}
& \left\|\left[\boldsymbol{u}_{h}-\tilde{\boldsymbol{u}}_{h}, p_{h}-\tilde{p}_{h}\right]\right\|\left\|_{h}\right\|\left[\boldsymbol{v}_{h}, q_{h}\right]\|\|_{h} \\
& \lesssim B_{s}\left(\left[\boldsymbol{u}_{h}-\tilde{\boldsymbol{u}}_{h}, p_{h}-\tilde{p}_{h}\right],\left[\boldsymbol{v}_{h}, q_{h}\right]\right) \\
& =B_{s}\left(\left[\boldsymbol{u}-\tilde{\boldsymbol{u}}_{h}, p-\tilde{p}_{h}\right],\left[\boldsymbol{v}_{h}, q_{h}\right]\right) \\
& \lesssim \\
& \quad \nu \varepsilon_{1}(\boldsymbol{u})\left\|\nabla \boldsymbol{v}_{h}\right\|+\sigma \varepsilon_{0}(\boldsymbol{u})\left\|\boldsymbol{v}_{h}\right\|+\sigma \ell_{p}^{2} \varepsilon_{0}(\nabla \cdot \boldsymbol{u})\left\|\nabla \cdot \boldsymbol{v}_{h}\right\| \\
& \quad+\frac{h^{2}}{\nu+\sigma \ell_{u}^{2}}\left(\nu \frac{\varepsilon_{1}(\boldsymbol{u})}{h}+\sigma \varepsilon_{0}(\boldsymbol{u})+\varepsilon_{1}(p)\right)\left(\nu \frac{C_{\mathrm{inv}}}{h}\left\|\nabla \boldsymbol{v}_{h}\right\|+\sigma\left\|\boldsymbol{v}_{h}\right\|+\sum_{K}\left\|\nabla q_{h}\right\|_{K}\right) \\
& \quad-\left(p-\tilde{p}_{h}, \nabla \cdot \boldsymbol{v}_{h}\right)+\left(q_{h}, \nabla \cdot\left(\boldsymbol{u}-\tilde{\boldsymbol{u}}_{h}\right)\right. \\
& \quad+\tau_{f} \sum_{E}\left\langle\llbracket \boldsymbol{n}\left(p-\tilde{p}_{h}\right)-\nu \partial_{h}\left(\boldsymbol{u}-\tilde{\boldsymbol{u}}_{h}\right) \rrbracket, \llbracket \boldsymbol{n} q_{h}+\nu \partial_{n} \boldsymbol{v}_{h} \rrbracket\right\rangle_{E} \\
& \quad \lesssim E(h)\left\|\left[\boldsymbol{v}_{h}, q_{h}\right]\right\| h-\left(p-\tilde{p}_{h}, \nabla \cdot \boldsymbol{v}_{h}\right)+\left(q_{h}, \nabla \cdot\left(\boldsymbol{u}-\tilde{\boldsymbol{u}}_{h}\right)\right. \\
& (3.28) \\
& \quad+\tau_{f} \sum_{E}\left\langle\llbracket \boldsymbol{n}\left(p-\tilde{p}_{h}\right)-\nu \partial_{n}\left(\boldsymbol{u}-\tilde{\boldsymbol{u}}_{h}\right) \rrbracket, \llbracket \boldsymbol{n} q_{h}+\nu \partial_{h} \boldsymbol{v}_{h} \rrbracket\right\rangle_{E} .
\end{aligned}
$$

The terms that remain to be expressed in terms of $E(h)$ and $\left\|\left[\boldsymbol{v}_{h}, q_{h}\right]\right\|_{h}$ can be bounded as follows:

$$
\begin{aligned}
-\left(p-\tilde{p}_{h}, \nabla \cdot \boldsymbol{v}_{h}\right) & \leq\left(\nu+\sigma \ell_{p}^{2}\right)^{1 / 2}\left\|\nabla \cdot \boldsymbol{v}_{h}\right\|\left(\nu+\sigma \ell_{u}^{2}\right)^{-1 / 2} h \varepsilon_{1}(p) \\
& \leq E(h)\left\|\left[\boldsymbol{v}_{h}, q_{h}\right]\right\|_{h},
\end{aligned}
$$

where we have assumed that $\ell_{p} \geq \ell_{u}$. Likewise,

$$
\begin{aligned}
& \left(q_{h}, \nabla \cdot\left(\boldsymbol{u}-\tilde{\boldsymbol{u}}_{h}\right)\right)=-\sum_{K}\left\langle\nabla q_{h}, \boldsymbol{u}-\tilde{\boldsymbol{u}}_{h}\right\rangle_{K}+\sum_{E}\left\langle\llbracket \boldsymbol{n} q_{h} \rrbracket, \boldsymbol{u}-\tilde{\boldsymbol{u}}_{h}\right\rangle_{E} \\
& \leq \sum_{K}\left(\nu+\sigma \ell_{u}^{2}\right)^{-1 / 2} h\left\|\nabla q_{h}\right\|_{K}\left(\nu+\sigma \ell_{p}^{2}\right)^{1 / 2} h^{-1}\left\|\boldsymbol{u}-\tilde{\boldsymbol{u}}_{h}\right\|_{K} \\
& \quad+\sum_{E}\left(\nu+\sigma \ell_{u}^{2}\right)^{-1 / 2} h^{1 / 2}\left\|\llbracket \boldsymbol{n} q_{h} \rrbracket\right\|_{E}\left(\nu+\sigma \ell_{p}^{2}\right)^{1 / 2} h^{-1 / 2}\left\|\boldsymbol{u}-\tilde{\boldsymbol{u}}_{h}\right\|_{E} \\
& \quad \lesssim E(h)\left\|\left[\boldsymbol{v}_{h}, q_{h}\right]\right\|_{h} .
\end{aligned}
$$

Finally,

$$
\tau_{f} \sum_{E}\left\langle\llbracket \boldsymbol{n}\left(p-\tilde{p}_{h}\right)-\nu \partial_{n}\left(\boldsymbol{u}-\tilde{\boldsymbol{u}}_{h}\right) \rrbracket, \llbracket \boldsymbol{n} q_{h}+\nu \partial_{n} \boldsymbol{v}_{h} \rrbracket\right\rangle_{E} \lesssim \hat{E}(h) \hat{N}\left(\left[\boldsymbol{v}_{h}, q_{h}\right]\right),
$$

Copyright (? by SIAM. Unauthorized reproduction of this article is prohibited. 
where

$$
\begin{aligned}
\hat{E}(h)^{2} & :=\tau_{f} \sum_{E}\left(\left\|\llbracket \boldsymbol{n}\left(p-\tilde{p}_{h}\right) \rrbracket\right\|_{E}^{2}+\nu^{2}\left\|\llbracket \partial_{n}\left(\boldsymbol{u}-\tilde{\boldsymbol{u}}_{h}\right) \rrbracket\right\|_{E}^{2}\right) \\
& \lesssim \tau_{f} h \varepsilon_{1}^{2}(p)+\tau_{f} \nu^{2} h^{-1} \varepsilon_{1}^{2}(\boldsymbol{u}) \\
& \lesssim E(h)^{2}, \\
\left.\hat{N}\left(\left[\boldsymbol{v}_{h}, q_{h}\right)\right]\right)^{2} & :=\tau_{f} \sum_{E}\left(\left\|\llbracket \boldsymbol{n} q_{h} \rrbracket\right\|_{E}^{2}+\nu^{2}\left\|\llbracket \partial_{n} \boldsymbol{v}_{h} \rrbracket\right\|_{E}^{2}\right) \\
& \lesssim \tau_{f} \sum_{E}\left\|\llbracket \boldsymbol{n} q_{h} \rrbracket\right\|_{E}^{2}+\tau_{f} \nu^{2} h^{-1}\left\|\nabla \boldsymbol{v}_{h}\right\|^{2} \\
& \lesssim\left\|\left[\boldsymbol{v}_{h}, q_{h}\right]\right\|_{h}^{2} .
\end{aligned}
$$

From (3.28)-(3.31) it follows that $\left\|\left[\boldsymbol{u}_{h}-\tilde{\boldsymbol{u}}_{h}, p_{h}-\tilde{p}_{h}\right]\right\|_{h} \lesssim E(h)$. This, (3.27), and the triangle inequality imply the result.

Remark 3.2. Consider for simplicity continuous pressure interpolation, and let us write the error estimate obtained as

$$
\begin{gathered}
\nu\left\|\nabla \boldsymbol{e}_{u}\right\|^{2}+\sigma\left\|\boldsymbol{e}_{u}\right\|^{2}+\sigma \ell_{p}^{2}\left\|\nabla \cdot \boldsymbol{e}_{u}\right\|^{2}+\frac{1}{\nu+\sigma L_{0}^{2}}\left\|e_{p}\right\|^{2}+\frac{h^{2}}{\nu+\sigma \ell_{u}^{2}}\left\|\nabla e_{p}\right\|^{2} \\
\lesssim\left(\nu+\sigma \ell_{p}^{2}\right) h^{2 k}\|\boldsymbol{u}\|_{k+1}^{2}+\sigma h^{2 k+2}\|\boldsymbol{u}\|_{k+1}^{2}+\frac{1}{\nu+\sigma \ell_{u}^{2}} h^{2 l+2}\|p\|_{l+1}^{2}
\end{gathered}
$$

where $\boldsymbol{e}_{u}=\boldsymbol{u}-\boldsymbol{u}_{h}$ and $e_{p}=p-p_{h}, k$ is the order of the velocity interpolation, $l$ the order of the pressure interpolation, and we have used the abbreviation $\|v\|_{i} \equiv\|v\|_{H^{i}(\Omega)}$ for $v$ either $\boldsymbol{u}$ or $p$. It is seen that if $\nu>0$, the estimate is optimal. The error is driven by the error in the viscous term. It is also observed that optimal balance of errors is found when $k=l+1$. It would be reasonable to try to keep this optimal balance in the limit $\nu \rightarrow 0$, although this is by no means necessary, and as we shall see, what can be considered the best method for Darcy's problem does not satisfy this requirement. This issue is discussed in section 4 .

3.4. Analysis of the OSS method. We analyze now problem (3.7) with $B_{s}$ and $L_{s}$ given by (3.13) and (3.14), respectively. As we shall see, we recover essentially the same results as for the ASGS formulation.

THEOREM 3.3 (stability of the OSS method). Suppose that $\ell_{p} \leq \ell_{u}$ and that $c_{1}>2 C_{\mathrm{tr}}$ in the definition (3.10)-(3.12) of the stabilization parameters. Then, the stability condition (3.18) holds for the OSS method.

Proof. Taking $\left[\boldsymbol{v}_{h}, q_{h}\right]=\left[\boldsymbol{u}_{h}, p_{h}\right]$ we have

$$
\begin{aligned}
B_{s}\left(\left[\boldsymbol{u}_{h}, p_{h}\right],\left[\boldsymbol{u}_{h}, p_{h}\right]\right)= & \nu\left\|\nabla \boldsymbol{u}_{h}\right\|^{2}+\sigma\left\|\boldsymbol{u}_{h}\right\|^{2} \\
& +\tau_{p} \sum_{K}\left\|P^{\perp}\left(\nabla \cdot \boldsymbol{u}_{h}\right)\right\|_{K}^{2}+\tau_{u} \sum_{K}\left\|P^{\perp}\left(\nabla p_{h}\right)\right\|_{K}^{2} \\
& +\tau_{f} \sum_{E}\left\|\llbracket \boldsymbol{n} p_{h} \rrbracket\right\|_{E}^{2}-\tau_{f} \nu^{2} \sum_{E}\left\|\llbracket \partial_{n} \boldsymbol{u}_{h} \rrbracket\right\|_{E}^{2} .
\end{aligned}
$$

Copyright (c) by SIAM. Unauthorized reproduction of this article is prohibited. 
Since $\tau_{f} \nu^{2} \sum_{E}\left\|\llbracket \partial_{n} \boldsymbol{u}_{h} \rrbracket\right\|_{E}^{2} \leq 2 \tau_{f} \nu \frac{C_{\mathrm{tr}}}{h} \nu\left\|\nabla \boldsymbol{u}_{h}\right\|^{2}$, the last term in (3.32) can be absorbed by the first using condition $c_{1}>2 C_{\mathrm{tr}}$.

Consider now $\boldsymbol{v}_{1}=\tau_{u} P\left(\nabla p_{h}\right), q_{1}=\tau_{p} P\left(\nabla \cdot \boldsymbol{u}_{h}\right)$. We have

$$
\begin{aligned}
& B_{s}\left(\left[\boldsymbol{u}_{h}, p_{h}\right],\left[\boldsymbol{v}_{1}, q_{1}\right]\right) \\
& \geq-\nu\left\|\nabla \boldsymbol{u}_{h}\right\| \frac{C_{\mathrm{inv}}}{h} \tau_{u}\left\|P\left(\nabla p_{h}\right)\right\| \\
&-\sigma\left\|\boldsymbol{u}_{h}\right\| \tau_{u}\left\|P\left(\nabla p_{h}\right)\right\| \\
&-\tau_{p} \sum_{K}\left\|P^{\perp}\left(\nabla \cdot \boldsymbol{u}_{h}\right)\right\|_{K} \frac{C_{\mathrm{inv}}}{h} \tau_{u}\left\|P\left(\nabla p_{h}\right)\right\|_{K} \\
&-\tau_{u} \sum_{K}\left\|P^{\perp}\left(\nabla p_{h}\right)\right\|_{K} \frac{C_{\mathrm{inv}}}{h} \tau_{p}\left\|P\left(\nabla \cdot \boldsymbol{u}_{h}\right)\right\|_{K} \\
&+\tau_{u} \sum_{K}\left\|P\left(\nabla p_{h}\right)\right\|_{K}^{2}+\tau_{p} \sum_{K}\left\|P\left(\nabla \cdot \boldsymbol{u}_{h}\right)\right\|_{K}^{2} \\
&+\tau_{f} \sum_{E}\left\langle\llbracket \boldsymbol{n} p_{h}-\nu \partial_{n} \boldsymbol{u}_{h} \rrbracket, \llbracket \tau_{p} \boldsymbol{n} P\left(\nabla \cdot \boldsymbol{u}_{h}\right)+\tau_{u} \nu \partial_{n} P\left(\nabla p_{h}\right) \rrbracket\right\rangle_{E},
\end{aligned}
$$

where we have used the inverse estimate (3.1).

Similarly to the proof of Theorem 3.1, using repeatedly Young's inequality we obtain

$$
\begin{aligned}
& B_{s}\left(\left[\boldsymbol{u}_{h}, p_{h}\right],\left[\boldsymbol{v}_{1}, q_{1}\right]\right) \\
& \quad \geq \tau_{u}\left[1-\tau_{u}\left(\nu \frac{C_{\mathrm{inv}}^{2}}{\beta_{1} h^{2}}+\frac{\sigma}{\beta_{2}}+\frac{C_{\mathrm{inv}}^{2}}{\beta_{3} h^{2}} \tau_{p}\right)\right]\left\|P\left(\nabla p_{h}\right)\right\|^{2} \\
& \quad+\tau_{p}\left[1-\tau_{p} \frac{C_{\mathrm{inv}}^{2}}{\beta_{4} h^{2}} \tau_{u}\right] \sum_{K}\left\|P\left(\nabla \cdot \boldsymbol{u}_{h}\right)\right\|_{K}^{2} \\
& \quad-C\left(\beta_{i}\right)\left(\nu\left\|\nabla \boldsymbol{u}_{h}\right\|^{2}+\sigma\left\|\boldsymbol{u}_{h}\right\|^{2}+\tau_{u} \sum_{K}\left\|P^{\perp}\left(\nabla p_{h}\right)\right\|_{K}^{2}+\tau_{p} \sum_{K}\left\|P^{\perp}\left(\nabla \cdot \boldsymbol{u}_{h}\right)\right\|_{K}^{2}\right) \\
& \quad+\tau_{f} \sum_{E}\left\langle\llbracket \boldsymbol{n} p_{h}-\nu \partial_{n} \boldsymbol{u}_{h} \rrbracket, \llbracket \tau_{p} \boldsymbol{n} P\left(\nabla \cdot \boldsymbol{u}_{h}\right)+\tau_{u} \nu \partial_{n} P\left(\nabla p_{h}\right) \rrbracket\right\rangle_{E},
\end{aligned}
$$

where $C\left(\beta_{i}\right)$ is a positive constant that depends on $\beta_{i}, i=1,2,3,4$. It remains to bound the term involving the interior boundaries, which can be done as follows:

$$
\begin{aligned}
\tau_{f} & \sum_{E}\left\langle\llbracket \boldsymbol{n} p_{h}-\nu \partial_{n} \boldsymbol{u}_{h} \rrbracket, \llbracket \tau_{p} \boldsymbol{n} P\left(\nabla \cdot \boldsymbol{u}_{h}\right)+\tau_{u} \nu \partial_{n} P\left(\nabla p_{h}\right) \rrbracket\right\rangle_{E} \\
& \gtrsim-N_{1}^{1 / 2}\left(\boldsymbol{u}_{h}, p_{h}\right) N_{2}^{1 / 2}\left(\boldsymbol{u}_{h}, p_{h}\right),
\end{aligned}
$$

Copyright $\odot$ by SIAM. Unauthorized reproduction of this article is prohibited. 
where

$$
\begin{aligned}
N_{1}\left(\boldsymbol{u}_{h}, p_{h}\right): & =\tau_{f} \sum_{E}\left\|\llbracket \boldsymbol{n} p_{h} \rrbracket\right\|_{E}^{2}+\tau_{f} \sum_{E}\left\|\llbracket \nu \partial_{n} \boldsymbol{u}_{h} \rrbracket\right\|_{E}^{2} \\
\leq & \tau_{f} \sum_{E}\left\|\llbracket \boldsymbol{n} p_{h} \rrbracket\right\|_{E}^{2}+\tau_{f} 2 \nu^{2} C_{\mathrm{tr}} h^{-1} \sum_{K}\left\|\nabla \boldsymbol{u}_{h}\right\|_{K}^{2} \\
\lesssim & \tau_{f} \sum_{E}\left\|\llbracket \boldsymbol{n} p_{h} \rrbracket\right\|_{E}^{2}+\nu\left\|\nabla \boldsymbol{u}_{h}\right\|^{2} \\
N_{2}\left(\boldsymbol{u}_{h}, p_{h}\right):= & \tau_{f} \sum_{E}\left\|\llbracket \boldsymbol{n} \tau_{p} P\left(\nabla \cdot \boldsymbol{u}_{h}\right) \rrbracket\right\|_{E}^{2}+\tau_{f} \sum_{E}\left\|\llbracket \nu \tau_{u} \partial_{n} P\left(\nabla p_{h}\right) \rrbracket\right\|_{E}^{2} \\
\leq & 2 C_{\mathrm{tr}} h^{-1} \tau_{f} \tau_{p}^{2} \sum_{K}\left\|P\left(\nabla \cdot \boldsymbol{u}_{h}\right)\right\|_{K}^{2} \\
& +2 C_{\mathrm{tr}} h^{-1} C_{\mathrm{inv}}^{2} h^{-2} \nu^{2} \tau_{f} \tau_{u}^{2} \sum_{K}\left\|P\left(\nabla p_{h}\right)\right\|_{K}^{2} \\
\lesssim & \tau_{p} \sum_{K}\left\|P\left(\nabla \cdot \boldsymbol{u}_{h}\right)\right\|_{K}^{2}+\tau_{u} \sum_{K}\left\|P\left(\nabla p_{h}\right)\right\|_{K}^{2} .
\end{aligned}
$$

We have used that $\tau_{f} \tau_{p} \lesssim h$. From (3.34) we have

$$
\begin{aligned}
\tau_{f} & \sum_{E}\left\langle\llbracket \boldsymbol{n} p_{h}-\nu \partial_{n} \boldsymbol{u}_{h} \rrbracket, \llbracket \tau_{p} \boldsymbol{n} P\left(\nabla \cdot \boldsymbol{u}_{h}\right)+\tau_{u} \nu \partial_{n} P\left(\nabla p_{h}\right) \rrbracket\right\rangle_{E} \\
& \gtrsim-\tau_{f} \sum_{E}\left\|\llbracket \boldsymbol{n} p_{h} \rrbracket\right\|_{E}^{2}-\nu\left\|\nabla \boldsymbol{u}_{h}\right\|^{2} \\
& -\frac{1}{\beta_{4}}\left(\tau_{p} \sum_{K}\left\|P\left(\nabla \cdot \boldsymbol{u}_{h}\right)\right\|_{K}^{2}+\tau_{u} \sum_{K}\left\|P\left(\nabla p_{h}\right)\right\|_{K}^{2}\right) .
\end{aligned}
$$

It is seen from (3.33) that for $\beta_{i}, i=1,2,3,4$, sufficiently large everything works provided $\tau_{u} \tau_{p} \lesssim h^{2}$, which follows from condition $\ell_{p} \leq \ell_{u}$ (in terms of $h$ ).

The only term missing from $\|\cdot\|_{h}^{2}$ is the $L^{2}$-norm of the pressure. It can be included as for the ASGS method.

For simplicity, convergence will be proved when $\boldsymbol{f}$ and $g$ are finite element functions, although the extension to the general case is easy.

ThEOREM 3.4 (convergence of the OSS method). Under the same conditions as in Theorem 3.3, assume also that $\boldsymbol{f}$ and $g$ are finite element functions and that $\ell_{u}=\ell_{p}$. Then the error estimate (3.26) holds for the OSS method.

Proof. The proof is almost the same as that of Theorem 3.2. Let us start noting that

$$
\begin{array}{r}
\left\|\left[\boldsymbol{u}_{h}-\tilde{\boldsymbol{u}}_{h}, p_{h}-\tilde{p}_{h}\right]\right\|\|\|_{h}\left\|\left[\boldsymbol{v}_{h}, q_{h}\right]\right\| \|_{h} \leq B_{s}\left(\left[\boldsymbol{u}_{h}-\tilde{\boldsymbol{u}}_{h}, p_{h}-\tilde{p}_{h}\right],\left[\boldsymbol{v}_{h}, q_{h}\right]\right) \\
\quad=B_{s}\left(\left[\boldsymbol{u}-\tilde{\boldsymbol{u}}_{h}, p-\tilde{p}_{h}\right],\left[\boldsymbol{v}_{h}, q_{h}\right]\right)+B_{s}\left(\left[\boldsymbol{u}_{h}-\boldsymbol{u}, p_{h}-p\right],\left[\boldsymbol{v}_{h}, q_{h}\right]\right) .
\end{array}
$$

The first term in (3.35) has a slightly simpler expression than in Theorem 3.2:

$$
\begin{aligned}
& B_{s}\left(\left[\boldsymbol{u}-\tilde{\boldsymbol{u}}_{h}, p-\tilde{p}_{h}\right],\left[\boldsymbol{v}_{h}, q_{h}\right]\right) \lesssim \nu \varepsilon_{1}(\boldsymbol{u})\left\|\nabla \boldsymbol{v}_{h}\right\|+\sigma \varepsilon_{0}(\boldsymbol{u})\left\|\boldsymbol{v}_{h}\right\|+\sigma \ell_{p}^{2} \varepsilon_{0}(\nabla \cdot \boldsymbol{u})\left\|\nabla \cdot \boldsymbol{v}_{h}\right\| \\
& \quad+\frac{h^{2}}{\nu+\sigma \ell_{u}^{2}} \varepsilon_{1}(p)\left\|\nabla q_{h}\right\|-\left(p-\tilde{p}_{h}, \nabla \cdot \boldsymbol{v}_{h}\right)+\left(q_{h}, \nabla \cdot\left(\boldsymbol{u}-\tilde{\boldsymbol{u}}_{h}\right)\right) \\
& \quad+\tau_{f} \sum_{E}\left\langle\llbracket \boldsymbol{n}\left(p-\tilde{p}_{h}\right)-\nu \partial_{n}\left(\boldsymbol{u}-\tilde{\boldsymbol{u}}_{h}\right) \rrbracket, \llbracket \boldsymbol{n} q_{h}+\nu \partial_{h} \boldsymbol{v}_{h} \rrbracket\right\rangle_{E},
\end{aligned}
$$

Copyright $\odot$ by SIAM. Unauthorized reproduction of this article is prohibited. 
that can be bounded as (3.28). We may continue as in the proof of Theorem 3.2, except that now we have a consistency error given by the second term in (3.35) that can be bounded as follows:

$$
\begin{aligned}
B_{s}\left(\left[\boldsymbol{u}-\boldsymbol{u}_{h}, p-p_{h}\right],\left[\boldsymbol{v}_{h}, q_{h}\right]\right) & =\tau_{u} \sum_{K}\left\langle P^{\perp}(\sigma \boldsymbol{u}+\nabla p), \nabla q_{h}\right\rangle_{K} \\
& =\tau_{u} \sum_{K}\left\langle P^{\perp}(\nu \Delta \boldsymbol{u}), \nabla q_{h}\right\rangle_{K} \\
& \lesssim \sqrt{\tau_{u}} \sum_{K} \sqrt{\nu} h\|\Delta \boldsymbol{u}-P(\Delta \boldsymbol{u})\|_{K}\left\|\nabla q_{h}\right\|_{K} \\
& \lesssim \sqrt{\nu} \varepsilon_{1}(\boldsymbol{u}) \sqrt{\tau_{u}} \sum_{K}\left\|\nabla q_{h}\right\|_{K} \\
& \lesssim E(h)\left\|\left[\boldsymbol{v}_{h}, q_{h}\right]\right\|_{h},
\end{aligned}
$$

where we have used the best approximation property of $P$ and the fact that $P^{\perp}\left(\nabla p_{h}\right)$ can be replaced by $P^{\perp}\left(\sigma \boldsymbol{u}_{h}+\nabla p_{h}\right)$ in the definition of $B_{s}$ without changing the method.

4. Duality arguments and convergence estimates for Darcy's problem. So far we have considered the Stokes-Darcy problem. Apart from the unified treatment we have introduced, the stabilized formulations we propose are nonstandard in the case $\nu=0$ because of the effect of the length scales $\ell_{p}$ and $\ell_{u}$ introduced in the stabilization parameters (3.10) and (3.11). Therefore, in this section we consider in detail the Darcy problem and, moreover, derive improved error estimates by using duality arguments.

The error estimate obtained in Theorems 3.2 and 3.4 in the case $\nu=0$ can be written as

$$
\begin{aligned}
& \sigma\left\|\boldsymbol{e}_{u}\right\|^{2}+\sigma \ell_{p}^{2}\left\|\nabla \cdot \boldsymbol{e}_{u}\right\|^{2}+\frac{1}{\sigma L_{0}^{2}}\left\|e_{p}\right\|^{2}+\frac{h^{2}}{\sigma \ell_{u}^{2}} \sum_{K}\left\|\nabla e_{p}\right\|_{K}^{2}+\frac{h}{\sigma \ell_{u}^{2}} \sum_{E}\left\|\llbracket \boldsymbol{n} e_{p} \rrbracket\right\|_{E}^{2} \\
& \lesssim \sigma \ell_{p}^{2} \varepsilon_{1}^{2}(\boldsymbol{u})+\sigma \varepsilon_{0}^{2}(\boldsymbol{u})+\frac{1}{\sigma \ell_{u}^{2}} \varepsilon_{0}^{2}(p) \\
& \lesssim \sigma \ell_{p}^{2} h^{2 k}\|\boldsymbol{u}\|_{k+1}^{2}+\sigma h^{2 k+2}\|\boldsymbol{u}\|_{k+1}^{2}+\frac{1}{\sigma \ell_{u}^{2}} h^{2 l+2}\|p\|_{l+1}^{2} .
\end{aligned}
$$

In order to obtain improved error estimates in $L^{2}(\Omega)$ for $\boldsymbol{e}_{u}$ and $e_{p}$ we need to assume that the adjoint problem

$$
\begin{aligned}
\boldsymbol{w}-\nabla \xi=\boldsymbol{f} & \text { in } \Omega, \\
-\nabla \cdot \boldsymbol{w}=g & \text { in } \Omega, \\
\boldsymbol{n} \cdot \boldsymbol{w}=0 & \text { on } \partial \Omega,
\end{aligned}
$$

satisfies the elliptic regularity assumption

$$
\begin{array}{ll}
\|\xi\|_{2} \lesssim\|g\|+\|\nabla \cdot \boldsymbol{f}\| & \text { in all cases, } \\
\|\boldsymbol{w}\|_{1} \lesssim\|g\| & \text { if } \boldsymbol{f}=\mathbf{0},
\end{array}
$$

together with the obvious general stability estimate

$$
\|\boldsymbol{w}\| \leq\|\boldsymbol{f}\| \quad \text { if } g=0 .
$$

It is known that (4.2)-(4.3) hold if $\Omega$ is convex and polyhedral or with twice differentiable boundary. 
4.1. Duality argument for the ASGS method. To simplify the notation, we will disregard in what follows the algorithmic constants $c_{2}^{u}$ and $c_{2}^{p}$. Only in the last step of the proof of the following theorem will we need to assume $c_{2}^{u}$ large enough. The bilinear form of the problem using the ASGS formulation can then be written as

$$
\begin{gathered}
B_{s}\left(\left[\boldsymbol{u}_{h}, p_{h}\right],\left[\boldsymbol{v}_{h}, q_{h}\right]\right)=B\left(\left[\boldsymbol{u}_{h}, p_{h}\right],\left[\boldsymbol{v}_{h}, q_{h}\right]\right)+\sigma \ell_{p}^{2} \sum_{K}\left\langle\nabla \cdot \boldsymbol{u}_{h}, \nabla \cdot \boldsymbol{v}_{h}\right\rangle_{K} \\
+\frac{h^{2}}{\sigma \ell_{u}^{2}} \sum_{K}\left\langle\sigma \boldsymbol{u}_{h}+\nabla p_{h},-\sigma \boldsymbol{v}_{h}+\nabla q_{h}\right\rangle_{K}+\frac{h}{\sigma \ell_{u}^{2}} \sum_{E}\left\langle\llbracket \boldsymbol{n} p_{h} \rrbracket, \llbracket \boldsymbol{n} q_{h} \rrbracket\right\rangle_{E},
\end{gathered}
$$

where now $B([\boldsymbol{u}, p],[\boldsymbol{v}, q])=\sigma(\boldsymbol{u}, \boldsymbol{v})-(p, \nabla \cdot \boldsymbol{v})+(q, \nabla \cdot \boldsymbol{u})$.

TheOrem 4.1. Assume the same conditions as in Theorem 3.2, now with $\nu=0$ and, moreover, assume (4.2)-(4.3) to hold. Furthermore, for $\ell_{u}=h$ and piecewise constant pressures $(l=0)$, we also require the constant $c_{2}^{u}$ in (3.11)-(3.12) to be large enough. Under these assumptions, there holds

$$
\begin{aligned}
\left\|\boldsymbol{e}_{u}\right\|^{2} & \lesssim\left(h^{2}+\frac{\ell_{p}^{4}}{L_{0}^{2}}+h^{2} \frac{\ell_{p}^{4}}{\ell_{u}^{4}}\right)\left\|\nabla \cdot \boldsymbol{e}_{u}\right\|^{2}+\frac{1}{\sigma^{2}}\left(\frac{h^{4}}{\ell_{u}^{4}}+\frac{h^{2}}{L_{0}^{2}}\right) \sum_{K}\left\|\nabla e_{p}\right\|_{K}^{2}, \\
\left\|e_{p}\right\|^{2} & \lesssim \sigma^{2} \ell_{p}^{4}\left\|\nabla \cdot \boldsymbol{e}_{u}\right\|^{2}+h^{2} \sum_{K}\left\|\nabla e_{p}\right\|_{K}^{2} .
\end{aligned}
$$

Proof. Consider the adjoint problem

$$
\begin{aligned}
\sigma \boldsymbol{w}-\nabla \xi & =\sigma \hat{\boldsymbol{e}}_{u}, \\
-\nabla \cdot \boldsymbol{w} & =\frac{1}{\sigma L_{0}^{2}} \hat{e}_{p} .
\end{aligned}
$$

We have

$$
\begin{aligned}
\sigma\left(\hat{\boldsymbol{e}}_{u}, \boldsymbol{e}_{u}\right)+ & \frac{1}{\sigma L_{0}^{2}}\left(\hat{e}_{p}, e_{p}\right) \\
= & -\left(e_{p}, \nabla \cdot \boldsymbol{w}\right)+\sigma\left(\boldsymbol{w}, \boldsymbol{e}_{u}\right)-\left(\nabla \xi, \boldsymbol{e}_{u}\right) \\
= & \sigma\left(\boldsymbol{e}_{u}, \boldsymbol{w}\right)-\left(e_{p}, \nabla \cdot \boldsymbol{w}\right)+\left(\xi, \nabla \cdot \boldsymbol{e}_{u}\right) \\
= & B\left(\left[\boldsymbol{e}_{u}, e_{p}\right],[\boldsymbol{w}, \xi]\right) \\
= & B\left(\left[\boldsymbol{e}_{u}, e_{p}\right],[\boldsymbol{w}, \xi]\right)+\sigma \ell_{p}^{2}\left(\nabla \cdot \boldsymbol{e}_{u}, \nabla \cdot \boldsymbol{w}\right)+\frac{\ell_{p}^{2}}{L_{0}^{2}}\left(\nabla \cdot \boldsymbol{e}_{u}, \hat{e}_{p}\right) \\
& +\frac{h^{2}}{\sigma \ell_{u}^{2}} \sum_{K}\left\langle\sigma \boldsymbol{e}_{u}+\nabla e_{p},-\sigma \boldsymbol{w}+\nabla \xi+\sigma \hat{\boldsymbol{e}}_{u}\right\rangle_{K} \\
& +\frac{h}{\sigma \ell_{u}^{2}} \sum_{E}\left\langle\llbracket \boldsymbol{n} e_{p} \rrbracket, \llbracket \boldsymbol{n} \xi \rrbracket\right\rangle_{E} \\
= & B_{s}\left(\left[\boldsymbol{e}_{u}, e_{p}\right],[\boldsymbol{w}, \xi]\right)+\frac{\ell_{p}^{2}}{L_{0}^{2}}\left(\nabla \cdot \boldsymbol{e}_{u}, \hat{e}_{p}\right)+\frac{h^{2}}{\sigma \ell_{u}^{2}} \sum_{K}\left\langle\sigma \boldsymbol{e}_{u}+\nabla e_{p}, \sigma \hat{\boldsymbol{e}}_{u}\right\rangle_{K} \\
= & B_{s}\left(\left[\boldsymbol{e}_{u}, e_{p}\right],\left[\boldsymbol{w}-\tilde{\boldsymbol{w}}_{h}, \xi-\tilde{\xi}_{h}\right]\right) \\
& +\frac{\ell_{p}^{2}}{L_{0}^{2}}\left(\nabla \cdot \boldsymbol{e}_{u}, \hat{e}_{p}\right)+\frac{h^{2}}{\sigma \ell_{u}^{2}} \sum_{K}\left\langle\sigma \boldsymbol{e}_{u}+\nabla e_{p}, \sigma \hat{\boldsymbol{e}}_{u}\right\rangle_{K}, \\
&
\end{aligned}
$$

Copyright $@$ by SIAM. Unauthorized reproduction of this article is prohibited. 
where $\tilde{\boldsymbol{w}}_{h}$ and $\tilde{\xi}_{h}$ are finite element functions to be defined. For $\tilde{\xi}_{h}$, we need to distinguish two different situations. First, we consider piecewise constant pressures $(l=0)$ and take $\tilde{\xi}_{h} \in Q_{h}$. Secondly, we consider the case $l>0$ and pick $\tilde{\xi}_{h} \in$ $Q_{h} \cap H^{1}(\Omega)$. Let us bound the first term:

$$
\begin{aligned}
& B_{s}\left(\left[\boldsymbol{e}_{u}, e_{p}\right],\left[\boldsymbol{w}-\tilde{\boldsymbol{w}}_{h}, \xi-\tilde{\xi}_{h}\right]\right) \lesssim \sigma\left\|\boldsymbol{e}_{u}\right\|\left\|\boldsymbol{w}-\tilde{\boldsymbol{w}}_{h}\right\|-\left(e_{p}, \nabla \cdot\left(\boldsymbol{w}-\tilde{\boldsymbol{w}}_{h}\right)\right) \\
& \quad+h\|\xi\|_{2}\left\|\boldsymbol{e}_{u}\right\|+\sigma \ell_{p}^{2}\left\|\nabla \cdot\left(\boldsymbol{w}-\tilde{\boldsymbol{w}}_{h}\right)\right\|\left\|\nabla \cdot \boldsymbol{e}_{u}\right\| \\
& \quad+\frac{h^{2}}{\sigma \ell_{u}^{2}} h\|\xi\|_{2} \sum_{K}\left\|\nabla e_{p}\right\|_{K}+\beta \frac{h}{\sigma \ell_{u}^{2}} h^{-1 / 2} h^{3 / 2}\|\xi\|_{2}\left\|e_{p}\right\|,
\end{aligned}
$$

where we have used (3.3) and (3.5) in order to obtain the last term when $l=0$; in this case $\beta=1$. For $l>0$, we may consider $\tilde{\xi}_{h} \in H^{1}(\Omega)$, and the previous inequality is true for $\beta=0$.

Suppose that $\hat{\boldsymbol{e}}_{u}=0, \hat{e}_{p}=e_{p}$. In this case we take as $\tilde{\boldsymbol{w}}_{h}$ an optimal interpolation of $\boldsymbol{w}$ in the finite element space. Using the shift assumptions (4.2)-(4.3) we get

$$
\begin{aligned}
\|\xi\|_{2} & \lesssim \frac{1}{L_{0}^{2}}\left\|e_{p}\right\|, \\
\left\|\boldsymbol{w}-\tilde{\boldsymbol{w}}_{h}\right\| & \leq h\|\boldsymbol{w}\|_{1} \leq \frac{h}{\sigma L_{0}^{2}}\left\|e_{p}\right\|, \\
-\left(e_{p}, \nabla \cdot\left(\boldsymbol{w}-\tilde{\boldsymbol{w}}_{h}\right)\right) & =\sum_{K}\left\langle\nabla e_{p}, \boldsymbol{w}-\tilde{\boldsymbol{w}}_{h}\right\rangle_{K} \leq \sum_{K}\left\|\nabla e_{p}\right\|_{K} \frac{h}{\sigma L_{0}^{2}}\left\|e_{p}\right\|_{K}, \\
\sigma \ell_{p}^{2}\left\|\nabla \cdot\left(\boldsymbol{w}-\tilde{\boldsymbol{w}}_{h}\right)\right\|\left\|\nabla \cdot \boldsymbol{e}_{u}\right\| & \leq \sigma \ell_{p}^{2} \frac{1}{\sigma L_{0}^{2}}\left\|e_{p}\right\|\left\|\nabla \cdot \boldsymbol{e}_{u}\right\| .
\end{aligned}
$$

Using these bounds in (4.10) and the result in (4.9), together with Schwartz's inequality in the second term in the right-hand side of (4.9), we obtain, after using repeatedly Young's inequality and considering the appropriate constants in the stabilization parameters,

$$
\frac{1}{\sigma L_{0}^{2}}\left\|e_{p}\right\|^{2} \lesssim \sigma \frac{h^{2}}{L_{0}^{2}}\left\|\boldsymbol{e}_{u}\right\|^{2}+\frac{h^{2}}{\sigma L_{0}^{2}} \sum_{K}\left\|\nabla e_{p}\right\|_{K}^{2}+\frac{\sigma \ell_{p}^{4}}{L_{0}^{2}}\left\|\nabla \cdot \boldsymbol{e}_{u}\right\|^{2}
$$

Note that the last term in (4.10) yields a term of the form $\frac{\beta C h^{2}}{c_{2}^{u} \sigma \ell_{u}^{2} L_{0}^{2}}\left\|e_{p}\right\|^{2}$ when constants are accounted for. When $\ell_{u}=h$ and $l=0$, this term can be absorbed by the one in the left-hand side when $c_{2}^{u}$ is large enough.

Suppose now that $\hat{\boldsymbol{e}}_{u}=\boldsymbol{e}_{u}, \hat{e}_{p}=0$. Let $\tilde{\xi}_{h}$ be an optimal finite element interpolant of $\xi$. Special care needs to be taken for the selection of $\tilde{\boldsymbol{w}}_{h}$. By taking as unknown $-\xi$ and changing the sign of (4.8), it is clear that (4.7)-(4.8) is a standard Darcy problem which we may approximate using the ASGS method with $\ell_{p}=L_{0}$. If $\tilde{\boldsymbol{w}}_{h}$ is the solution to this problem, it will satisfy

$$
\begin{aligned}
\left\|\nabla \cdot \tilde{\boldsymbol{w}}_{h}\right\| & \lesssim \frac{1}{L_{0}}\left\|\boldsymbol{e}_{u}\right\|, \\
\left\|\boldsymbol{w}-\tilde{\boldsymbol{w}}_{h}\right\| & \leq \varphi(h)\|\boldsymbol{w}\| \lesssim \varphi(h)\left\|\boldsymbol{e}_{u}\right\|,
\end{aligned}
$$

where $\varphi(h) \rightarrow 0$ as $h \rightarrow 0$ and (4.4) has been used in (4.13). Property (4.12) is a consequence of the stability result proved in Theorem 3.1, whereas (4.13) also follows 
from the a priori estimate resulting from Theorem 3.1 (since the solution to the ASGS formulation is bounded in the norm (3.15), there must be a weakly convergent subsequence that, using classical arguments, must converge in norm to $[\boldsymbol{w}, \xi]$ ). Observe that it is crucial to use $\ell_{p}=L_{0}$. In any case, the important point is the existence in the finite element space of a function $\tilde{\boldsymbol{w}}_{h}$ satisfying (4.12)-(4.13).

Using the shift assumption (4.2) and (4.12) we get

$$
\begin{aligned}
\|\xi\|_{2} & \lesssim \sigma\left\|\nabla \cdot \boldsymbol{e}_{u}\right\|, \\
-\left(e_{p}, \nabla \cdot\left(\boldsymbol{w}-\tilde{\boldsymbol{w}}_{h}\right)\right) & \leq\left\|e_{p}\right\|\left\|\nabla \cdot \tilde{\boldsymbol{w}}_{h}\right\| \lesssim\left\|e_{p}\right\| \frac{1}{L_{0}}\left\|\boldsymbol{e}_{u}\right\|, \\
\sigma \ell_{p}^{2}\left\|\nabla \cdot\left(\boldsymbol{w}-\tilde{\boldsymbol{w}}_{h}\right)\right\|\left\|\nabla \cdot \boldsymbol{e}_{u}\right\| & \lesssim \sigma \ell_{p}^{2} \frac{1}{L_{0}}\left\|\boldsymbol{e}_{u}\right\|\left\|\nabla \cdot \boldsymbol{e}_{u}\right\| .
\end{aligned}
$$

Once again, using these bounds in (4.10) and the result in (4.9), taking $h$ sufficiently small in (4.13), and applying Young's inequality we obtain

$$
\sigma\left\|\boldsymbol{e}_{u}\right\|^{2} \lesssim \frac{1}{\sigma}\left(\frac{1}{L_{0}^{2}}+\frac{h^{2}}{\ell_{u}^{4}}\right)\left\|e_{p}\right\|^{2}+\sigma\left(h^{2}+\frac{\ell_{p}^{4}}{L_{0}^{2}}\right)\left\|\nabla \cdot \boldsymbol{e}_{u}\right\|^{2}+\frac{h^{4}}{\sigma \ell_{u}^{4}} \sum_{K}\left\|\nabla e_{p}\right\|_{K}^{2}
$$

The theorem follows combining (4.11) and (4.14). When $\ell_{u}=h$ and $l=0$, we also need to assume that the constant $c_{2}^{u}$ is sufficiently large.

4.2. Duality argument for the OSS method. Now the bilinear form of the stabilized problem is given by

$$
\begin{aligned}
B_{s}\left(\left[\boldsymbol{u}_{h}, p_{h}\right],\left[\boldsymbol{v}_{h}, q_{h}\right]\right)= & B\left(\left[\boldsymbol{u}_{h}, p_{h}\right],\left[\boldsymbol{v}_{h}, q_{h}\right]\right)+\sigma \ell^{2} \sum_{K}\left\langle\nabla \cdot \boldsymbol{v}_{h}, P^{\perp}\left(\nabla \cdot \boldsymbol{u}_{h}\right)\right\rangle_{K} \\
& +\frac{h^{2}}{\sigma \ell^{2}} \sum_{K}\left\langle\nabla q_{h}, P^{\perp}\left(\nabla p_{h}\right)\right\rangle_{K}+\frac{h}{\sigma \ell^{2}} \sum_{E}\left\langle\llbracket \boldsymbol{n} p_{h} \rrbracket, \llbracket \boldsymbol{n} q_{h} \rrbracket\right\rangle_{E},
\end{aligned}
$$

where $\ell_{u}=\ell_{p}=\ell$ has been assumed, which is the assumption of Theorem 3.4.

TheOREM 4.2. Assume the same conditions as in Theorem 3.4 , now with $\nu=0$ and, moreover, assume (4.2)-(4.3) to hold. The same estimates (4.5)-(4.6) hold for the OSS method.

Proof. We proceed in a way similar to Theorem 4.1. Consider again problem (4.7)-(4.8). We have

$$
\begin{aligned}
\sigma\left(\hat{\boldsymbol{e}}_{u}, \boldsymbol{e}_{u}\right)+ & \frac{1}{\sigma L_{0}^{2}}\left(\hat{e}_{p}, e_{p}\right) \\
= & B\left(\left[\boldsymbol{e}_{u}, e_{p}\right],[\boldsymbol{w}, \xi]\right)+\frac{h}{\sigma \ell_{u}^{2}} \sum_{E}\left\langle\llbracket \boldsymbol{n} e_{p} \rrbracket, \llbracket \boldsymbol{n} \xi \rrbracket\right\rangle_{E} \\
= & B_{s}\left(\left[\boldsymbol{e}_{u}, e_{p}\right],[\boldsymbol{w}, \xi]\right)-\sigma \ell^{2}\left(\nabla \cdot \boldsymbol{w}, P^{\perp}\left(\nabla \cdot \boldsymbol{e}_{u}\right)\right)-\frac{h^{2}}{\sigma \ell^{2}}\left(\nabla \xi, P^{\perp}\left(\nabla e_{p}\right)\right) \\
= & B_{s}\left(\left[\boldsymbol{e}_{u}, e_{p}\right],\left[\boldsymbol{w}-\tilde{\boldsymbol{w}}_{h}, \xi-\tilde{\xi}_{h}\right]\right) \\
& -\sigma \ell^{2} \sum_{K}\left\langle\nabla \cdot \boldsymbol{w}-P(\nabla \cdot \boldsymbol{w}), P^{\perp}\left(\nabla \cdot \boldsymbol{e}_{u}\right)\right\rangle_{K} \\
& -\frac{h^{2}}{\sigma \ell^{2}} \sum_{K}\left\langle\nabla \xi-P(\nabla \xi), P^{\perp}\left(\nabla e_{p}\right)\right\rangle_{K} .
\end{aligned}
$$

Copyright (C) by SIAM. Unauthorized reproduction of this article is prohibited. 
For the first term in the right-hand side, the same bound (4.10) as for the ASGS method can be obtained. The second term can be bounded as

$$
\begin{aligned}
\sigma \ell^{2} \sum_{K}\left\langle\nabla \cdot \boldsymbol{w}-P(\nabla \cdot \boldsymbol{w}), P^{\perp}\left(\nabla \cdot \boldsymbol{e}_{u}\right)\right\rangle_{K} & \lesssim \sigma \ell^{2} h \sum_{K}\|\nabla \nabla \cdot \boldsymbol{w}\|_{K}\left\|\nabla \cdot \boldsymbol{e}_{u}\right\|_{K} \\
& \lesssim \frac{\ell^{2}}{L_{0}^{2}} h \sum_{K}\left\|\nabla \hat{e}_{p}\right\|_{K}\left\|\nabla \cdot \boldsymbol{e}_{u}\right\|_{K} .
\end{aligned}
$$

Finally,

$$
\frac{h^{2}}{\sigma \ell^{2}} \sum_{K}\left\langle\nabla \xi-P(\nabla \xi), P^{\perp}\left(\nabla e_{p}\right)\right\rangle_{K} \lesssim \frac{h^{2}}{\sigma \ell^{2}} h\|\xi\|_{2} \sum_{K}\left\|\nabla e_{p}\right\|_{K}
$$

From (4.15) and (4.16) we can proceed exactly as in the proof of Theorem 4.1 (in fact, with less terms to bound).

4.3. Accuracy of the stabilized formulations for Darcy's problem. When $\nu>0$, the accuracy of the stabilized formulations we have introduced has been discussed in Remark 3.2. The results obtained are optimal, as expected. However, the situation is more interesting when $\nu=0$. The general error estimate is (4.1), and the improved $L^{2}$-error estimates are given in (4.5)-(4.6). Depending on the expression of the length scales $\ell_{u}$ and $\ell_{p}$, we may obtain different convergence rates. From the inspection of the convergence estimates it follows that the cases that need to be taken into account are $\ell_{u}, \ell_{p}=h, L_{0},\left(h L_{0}\right)^{1 / 2}$. The rates of convergence obtained, as dictated by (4.1) and (4.5)-(4.6), have been collected in Table 4.1. Constants (depending on the continuous unknowns) multiplying $h$ have been omitted, in order to highlight the order of convergence. We have marked with an asterisk those conditional convergence results that hold only for $c_{2}^{u}$ sufficiently large when piecewise constant pressures are considered. The contribution to the error due to the velocity and the pressure interpolations has been explicitly displayed. The errors termed "original" are those resulting from (4.1), whereas those referred to as "via duality" are obtained from (4.5)-(4.6).

Several remarks need to be made from the observation of Table 4.1:

1. The term "optimal" or "suboptimal" qualifying each result refers to the case $k=l$, that is to say, when equal velocity-pressure interpolation is used.

2. Some suboptimal error estimates can be made optimal with the duality arguments of Theorems 4.1 and 4.2, which apply for all $k$ and $l$. It is worth noting that no improvement is obtained if $\ell_{p}=L_{0}$.

3. Suboptimal estimates can be made optimal with the choice of $k$ and $l$ indicated in the last row.

4. $\ell_{p}=\ell_{u}=h$ (method A) is the "classical" stabilized method. Its behavior is the expected one if Darcy's problem is understood as a mixed formulation of Poisson's problem.

5. $\ell_{p}=L_{0}, \ell_{u}=h$ (method B) has the best stability (the same as the continuous problem; see Remark 3.1). Remember, however, that our analysis does not apply in this case when the OSS method is used (method B applies only for the ASGS formulation). In any case, method $\mathrm{C}$ below exhibits better convergence rates for any choice of $k$ and $l$.

6. $\ell_{p}=\ell_{u}=\left(L_{0} h\right)^{1 / 2}$ (method $\mathrm{C}$ ) is the most accurate when equal velocitypressure interpolation is used. In spite of the apparently ad hoc choice of the length scales, let us recall that it can be motivated by scaling the original 
TABLE 4.1

Convergence rates according to the choice of the length scale in the stabilization parameters when $\nu=0$.

\begin{tabular}{|c||c|c|c|c|}
\hline $\begin{array}{c}\text { Method } \\
\ell_{p}, \ell_{u}=\end{array}$ & A (OSS, ASGS) & B (ASGS) & C (OSS, ASGS) & D (OSS, ASGS) \\
\hline$\left\|e_{u}\right\|$ & $h^{k+1}+h^{l}$ & $h^{k}+h^{l}$ & $h^{k+1 / 2}+h^{l+1 / 2}$ & $h^{k}+h^{l+1}$ \\
Original & Suboptimal & Suboptimal & Quasi-optimal & Suboptimal \\
\hline$\left\|e_{u}\right\|$ & $h^{k+1}+h^{l}$ & $h^{k}+h^{l}$ & $h^{k+1}+h^{l+1}$ & $h^{k}+h^{l+1}$ \\
Via duality & Suboptimal & Suboptimal & Optimal & Suboptimal \\
\hline$\left\|e_{p}\right\|$ & $h^{k+1}+h^{l}$ & $h^{k}+h^{l}$ & $h^{k+1 / 2}+h^{l+1 / 2}$ & $h^{k}+h^{l+1}$ \\
Original & Suboptimal & Suboptimal & Quasi-optimal & Suboptimal \\
\hline$\left\|e_{p}\right\|$ & $h^{k+2}+h^{l+1}$ & $h^{k}+h^{l}$ & $h^{k+1}+h^{l+1}$ & $h^{k}+h^{l+1}$ \\
Via duality & Optimal $*$ & Suboptimal & Optimal & Suboptimal \\
\hline$\left\|\nabla \cdot e_{u}\right\|$ & $h^{k}+h^{l-1}$ & $h^{k}+h^{l}$ & $h^{k}+h^{l}$ & $h^{k}+h^{l+1}$ \\
& Suboptimal & Optimal & Optimal & Optimal \\
\hline$\left\|\nabla e_{p}\right\|$ & $h^{k+1}+h^{l}$ & $h^{k}+h^{l}$ & $h^{k}+h^{l}$ & $h^{k-1}+h^{l}$ \\
& Optimal & Optimal & Optimal & Suboptimal \\
\hline$k, l$ Optimal & $k+1=l$ & $k=l$ & $k=l$ & $k=l+1$ \\
\hline
\end{tabular}

equations (2.1)-(2.2) so as to define a dimensionally consistent norm of the forcing terms (see [2]).

7. $\ell_{p}=\ell_{u}=L_{0}$ (method D) seems interesting if Darcy's problem is solved as the limit when $\nu \rightarrow 0$ of the Stokes problem. In particular, method D and method $\mathrm{C}$ are the only methods that allow $l=0$ (obviously, considering discontinuous pressures), the former with optimal order of convergence.

From these remarks it is even possible to draw a recommendation. First, it is clear that if equal interpolation is used, method $\mathrm{C}$ is the optimal choice. If the Stokes-Darcy problem is used with $k=l+1$, method $\mathrm{D}$ should be chosen.

In [25] the authors consider the case $\ell_{u}=h$ and $\ell_{p}=0$, and they just mention in passing the possibility $\ell_{p}=h$. The results obtained are essentially those of method A in Table 4.1 (excluding optimality obtained through duality), although numerical experiments showed an unexpected superconvergence behavior. In [22] the method is extended to the discontinuous Galerkin approach, that is to say, with nonconforming velocity spaces (no comment is made about the possibility $\ell_{p}=h$ ). Once more, the results obtained are those of the first column in Table 4.1, now in accordance with the numerical experiments. In [11], we consider the general problem, including viscosity (and other terms) corresponding to $\ell_{u}=h$ and $\ell_{p}=h$. The choice of the constants in the stabilization parameters allows us to overcome boundary layers due to large quotients $\sigma L_{0}^{2} / \nu$, but does not allow us to take $\nu=0$. However, in light of the results of Table 4.1, method $\mathrm{A}$ is superseded by method $\mathrm{C}$ when equal velocity-pressure interpolation is used, which was the situation targeted in the cited references. Only if $k+1=l$ should method A be preferred.

5. The Stokes-Darcy coupling through interfaces. In this section, we briefly describe the application of the stabilized methods introduced previously to a heterogeneous problem coupling free flow with filtration flow through a porous medium. Let us consider a partition of the domain $\Omega$ into $\Omega^{S}$, filled by a fluid governed by the Stokes problem, and $\Omega^{D}$, covering the porous medium. The continuity conditions on the Stokes-Darcy interface $\Sigma$ are not obvious due to the different regularity of the velocities in both sides of $\Sigma$. The three boundary conditions that must 
be enforced on $\Sigma$ are

$$
\begin{aligned}
\boldsymbol{u}^{S} \cdot \boldsymbol{n} & =\boldsymbol{u}^{D} \cdot \boldsymbol{n}, \\
-p^{S}+\nu \partial_{n} \boldsymbol{u}^{S} \cdot \boldsymbol{n} & =-p^{D}, \\
\nu \partial_{n} \boldsymbol{u}^{S} \cdot \boldsymbol{t} & =-\frac{\alpha}{\sqrt{\sigma}} \boldsymbol{u}^{S} \cdot \boldsymbol{t},
\end{aligned}
$$

where $\boldsymbol{u}^{S}$ and $\boldsymbol{u}^{D}$ denote the Stokes and Darcy velocities, $p^{S}$ and $p^{D}$ denote the Stokes and Darcy pressures, $\boldsymbol{n}$ is the outer unit normal to $\Omega^{S}$, and $\boldsymbol{t}$ is a unit vector tangent to $\Sigma$. The parameter $\alpha$ is the slip rate coefficient. The transmission condition (5.1) enforces the continuity of normal velocities, (5.2) enforces the continuity of normal stresses, and (5.3) is the so-called Beavers-Joseph-Saffman condition (see [3, 28]). The last transmission condition is phenomenological and relates the tangential component of the normal stress (for the Stokes problem) with its tangential velocity on the interface; this is a Robin-type interface condition that allows the Stokes fluid to slip in contact with the porous medium.

For the Stokes subproblem, we introduce $V^{S} \times Q^{S}$ that consists of pairs $\left[\boldsymbol{v}^{S}, q^{S}\right] \in$ $H^{1}\left(\Omega^{S}\right)^{d} \times L^{2}\left(\Omega^{S}\right)$ such that $\boldsymbol{v}^{S}$ vanishes on $\partial \Omega^{S} \backslash \Sigma$. Analogously, $V^{D} \times Q^{D}$ is the set of functions $\left[\boldsymbol{v}^{D}, q^{D}\right] \in H\left(\operatorname{div}, \Omega^{D}\right)^{d} \times L^{2}\left(\Omega^{D}\right)$ such that $\boldsymbol{v}^{D} \cdot \boldsymbol{n}$ vanishes on $\partial \Omega^{S} \backslash \Sigma$. We use $B_{s}^{S}$ to denote the stabilized bilinear form in $\Omega^{S}$ for the Stokes subproblem (with $\sigma=0$ ) and $B_{s}^{D}$ to indicate the one for the Darcy subproblem (with $\nu=0$ ). Let $V_{h}^{S} \times Q_{h}^{S}$ and $V_{h}^{D} \times Q_{h}^{D}$ be appropriate finite element spaces to approximate $V^{S} \times Q^{S}$ and $V^{D} \times Q^{D}$, respectively. Using the previous formulation, the discrete Stokes subproblem consists of finding $\left[\boldsymbol{u}_{h}^{S}, p_{h}^{S}\right] \in V_{h}^{S} \times Q_{h}^{S}$ such that

$$
B_{s}^{S}\left(\left[\boldsymbol{u}_{h}^{S}, p_{h}^{S}\right],\left[\boldsymbol{v}_{h}^{S}, q_{h}^{S}\right]\right)=L^{S}\left(\left[\boldsymbol{v}_{h}^{S}, q_{h}^{S}\right]\right)+\int_{\partial \Omega \backslash \Sigma}\left(\nu \partial_{n} \boldsymbol{u}_{h}^{S}-p_{h}^{S} \boldsymbol{n}\right) \cdot \boldsymbol{v}_{h}^{S} \mathrm{~d} \Sigma
$$

for any $\left[\boldsymbol{v}_{h}^{S}, q_{h}^{S}\right] \in V_{h}^{S} \times Q_{h}^{S}$. In $\Omega^{D}$ we seek $\left[\boldsymbol{u}_{h}^{D}, p_{h}^{D}\right] \in V_{h}^{D} \times Q_{h}^{D}$ such that

$$
B_{s}^{D}\left(\left[\boldsymbol{u}_{h}^{D}, p_{h}^{D}\right],\left[\boldsymbol{v}_{h}^{D}, q_{h}^{D}\right]\right)=L^{D}\left(\left[\boldsymbol{v}_{h}^{D}, q_{h}^{D}\right]\right)-\int_{\partial \Omega \backslash \Sigma}\left(p_{h}^{D} \boldsymbol{n}\right) \cdot \boldsymbol{v}_{h}^{D} \mathrm{~d} \Sigma
$$

for any $\left[\boldsymbol{v}_{h}^{D}, q_{h}^{D}\right] \in V_{h}^{D} \times Q_{h}^{D}$. In order to have a well-posed problem, these two subproblems have to be supplemented with the transmission conditions (5.1)-(5.3).

Let us consider a weak evaluation of interface stresses in (5.2)-(5.3); the strong evaluation of stresses has been proved to spoil stability and accuracy (see [8]). It is easily seen that the coupled problem supplemented with the transmission conditions on the interface reads as follows: find $\left[\boldsymbol{u}_{h}^{S}, p_{h}^{S}\right] \in V_{h}^{S} \times Q_{h}^{S}$ and $\left[\boldsymbol{u}_{h}^{D}, p_{h}^{D}\right] \in V_{h}^{D} \times Q_{h}^{D}$ satisfying $\boldsymbol{u}_{h}^{S} \cdot \boldsymbol{n}=\boldsymbol{u}_{h}^{D} \cdot \boldsymbol{n}$ on $\Sigma$ and such that

$$
\begin{aligned}
B_{s}^{S} & \left(\left[\boldsymbol{u}_{h}^{S}, p_{h}^{S}\right],\left[\boldsymbol{v}_{h}^{S}, q_{h}^{S}\right]\right)+B_{s}^{D}\left(\left[\boldsymbol{u}_{h}^{D}, p_{h}^{D}\right],\left[\boldsymbol{v}_{h}^{D}, q_{h}^{D}\right]\right) \\
& +\frac{\alpha}{\sqrt{\sigma}}\left(\boldsymbol{u}_{h}^{S} \cdot \boldsymbol{t}, \boldsymbol{v}_{h}^{S} \cdot \boldsymbol{t}\right)_{\Sigma}=L^{S}\left(\left[\boldsymbol{v}_{h}^{S}, q_{h}^{S}\right]\right)+L^{D}\left(\left[\boldsymbol{v}_{h}^{D}, q_{h}^{D}\right]\right)
\end{aligned}
$$

for any $\left[\boldsymbol{v}_{h}^{S}, q_{h}^{S}\right] \in V_{h}^{S} \times Q_{h}^{S}$ and $\left[\boldsymbol{v}_{h}^{D}, q_{h}^{D}\right] \in V_{h}^{D} \times Q_{h}^{D}$ satisfying $\boldsymbol{v}_{h}^{S} \cdot \boldsymbol{n}=\boldsymbol{v}_{h}^{D} \cdot \boldsymbol{n}$ on $\Sigma$. The continuity of stresses has been imposed in a weak way using a particular choice of the test functions and the continuity of normal velocities enforced by the trial space. The interface term comes from (5.3).

At this point, the stability and convergence results obtained above can be extended to this case, with small modifications. In particular, inequality (3.2) has to be used in order to bound the new interpolation error related to the interface term in (5.4). 
TABLE 6.1

Experimental convergence rates for the ASGS method according to the choice of the length scale in the stabilization parameters when $\nu=0$. The $P 1 / P 1$ pair.

\begin{tabular}{|c|c|c|c|c|}
\hline $\begin{array}{l}\text { Method } \\
\ell_{p}, \ell_{u}=\end{array}$ & $\begin{array}{c}\mathrm{A} \\
h, h \\
\end{array}$ & $\begin{array}{c}\mathrm{B} \\
L_{0}, h \\
\end{array}$ & $\begin{array}{c}\mathrm{C} \\
L_{0}{ }^{1 / 2} h^{1 / 2}, L_{0}{ }^{1 / 2} h^{1 / 2}\end{array}$ & $\begin{array}{c}\mathrm{D} \\
L_{0}, L_{0} \\
\end{array}$ \\
\hline$\left\|e_{u}\right\|$ & $1.92(1)$ & $1.95(1)$ & $2.05(2)$ & $1.97(1)$ \\
\hline$\left\|e_{p}\right\|$ & $1.93(2)$ & $1.85(1)$ & $2.20(2)$ & $2.04(1)$ \\
\hline$\left\|\nabla \cdot \boldsymbol{e}_{u}\right\|$ & $1.30(-)$ & $1.94(1)$ & $1.43(1)$ & $1.43(1)$ \\
\hline$\left\|\nabla e_{p}\right\|$ & 1.99 (1) & $1.91(1)$ & $1.70(1)$ & $0.58(-)$ \\
\hline
\end{tabular}

6. Numerical testing. In this section we carry out some numerical experiments in order to check the theoretical convergence rates proved in sections 3 and 4 . We consider three kinds of problems: Darcy flow, Stokes flow, and a heterogeneous problem coupling Stokes and Darcy systems through interfaces. With regard to the numerical schemes, we have considered both the ASGS and the OSS techniques with all the possible choices of the stabilization parameters that have been analyzed previously.

We have used two finite element velocity-pressure interpolations. First, both velocity and pressure have been approximated using continuous piecewise linear finite element spaces, i.e., the $\mathrm{P} 1 / \mathrm{P} 1$ pair. Then, we have replaced the continuous interpolation for the pressure by a discontinuous piecewise constant interpolation.

All test problems are defined in the domain $\Omega \equiv(0,1) \times(0,1)$. We have considered structured and regular meshes. The family of finite element partitions used in the convergence analysis consist of 3200, 7200, and 12800 linear triangle elements.

The definition of the stabilization parameters in (3.10)-(3.12) include the algorithmic constants $c_{1}, c_{2}^{p}, c_{2}^{u}$, and a characteristic length $L_{0}$. Let us define $c_{2}^{u}=c_{2}$ and $c_{2}^{p}=\gamma c_{2}$. We have used $c_{1}=1, c_{2}=2$, and $L_{0}=0.1 \sqrt[d]{\text { meas }(\Omega)}$ in all cases. Based on numerical experimentation, we have taken $\gamma=1$ for methods $\mathrm{A}$ and $\mathrm{C}$ and $\gamma=0.1$ for methods B and D.

In order to evaluate the error introduced by the numerical approximations, we have solved test problems with analytical solutions, obtained with the appropriate choices of $\boldsymbol{f}, g$, and boundary conditions.

6.1. Darcy flow. Let us start the numerical experimentation for the limit case when $\nu=0$, i.e., the Darcy problem. We consider the following analytical solution:

$$
\boldsymbol{u}=(-2 \pi \cos (2 \pi x) \sin (2 \pi y),-2 \pi \sin (2 \pi x) \cos (2 \pi y)), \quad p=\sin (2 \pi x) \sin (2 \pi y) .
$$

This test has been extracted from [25]. The analytical solution is obtained for $\boldsymbol{f}=\mathbf{0}$; in Darcy flow applications $f$ is usually constant. On the other hand, only the normal component of the velocity is enforced on the boundary.

For the $\mathrm{P} 1 / \mathrm{P} 1$ finite element pair, we show the numerical order of convergence obtained using the ASGS method in Table 6.1 and using the OSS method in Table 6.2. The theoretical order of convergence is indicated in parenthesis and (-) is used when no convergence is expected. For this test, superconvergent results are obtained for $\left\|\boldsymbol{e}_{u}\right\|$ when using methods A and D. Method D exhibits superconvergence for $\left\|e_{p}\right\|$. Method A approximates $\left\|\nabla \cdot \boldsymbol{e}_{u}\right\|$ poorly, and method D has a low order of convergence for $\left\|\nabla e_{p}\right\|$; in fact, no order of convergence was expected from the numerical analysis in both cases. As commented above, method $\mathrm{C}$ is the best choice for $k=l$ (the case under consideration), but method B (thanks to superconvergence) is also very 
TABle 6.2

Experimental convergence rates for the OSS method according to the choice of the length scale in the stabilization parameters when $\nu=0$. The $P 1 / P 1$ pair.

\begin{tabular}{|c||c|c|c|}
\hline $\begin{array}{c}\text { Method } \\
\ell_{p}, \ell_{u}=\end{array}$ & $\begin{array}{c}\mathrm{A} \\
h, h\end{array}$ & $\mathrm{C}$ & $\mathrm{D}$ \\
\hline${ }_{0}{ }^{1 / 2} h^{1 / 2}, L_{0}{ }^{1 / 2} h^{1 / 2}$ & $L_{0}, L_{0}$ \\
\hline \hline$\left\|e_{u}\right\|$ & $1.85(1)$ & $2.20(2)$ & $1.99(1)$ \\
\hline$\left\|e_{p}\right\|$ & $1.90(2)$ & $2.23(2)$ & $2.04(1)$ \\
\hline$\left\|\nabla \cdot e_{u}\right\|$ & $0.78(-)$ & $1.68(1)$ & $1.43(1)$ \\
\hline$\left\|\nabla e_{p}\right\|$ & $1.60(1)$ & $1.48(1)$ & $0.53(-)$ \\
\hline
\end{tabular}

TABLE 6.3

Experimental value of the error norms for the mesh with 3200 linear triangle elements using the ASGS method according to the choice of the length scale in the stabilization parameters when $\nu=0$. The $P 1 / P 1$ pair.

\begin{tabular}{|c|c|c|c|c|}
\hline $\begin{array}{l}\text { Method } \\
\ell_{p}, \ell_{u}= \\
\end{array}$ & $\begin{array}{c}\mathrm{A} \\
h, h \\
\end{array}$ & $\begin{array}{c}\mathrm{B} \\
L_{0}, h \\
\end{array}$ & $\begin{array}{c}\mathrm{C} \\
L_{0}{ }^{1 / 2} h^{1 / 2}, L_{0}{ }^{1 / 2} h^{1 / 2}\end{array}$ & $\begin{array}{c}\mathrm{D} \\
L_{0}, L_{0} \\
\end{array}$ \\
\hline$\left\|e_{u}\right\|$ & 0.0205 & 0.0181 & 0.0112 & 0.0107 \\
\hline$\left\|e_{p}\right\|$ & 0.0206 & 0.0222 & 0.0207 & 0.0091 \\
\hline$\left\|\nabla \cdot \boldsymbol{e}_{u}\right\|$ & 0.6607 & 0.1758 & 0.0778 & 0.0371 \\
\hline$\left\|\nabla e_{p}\right\|$ & 0.0254 & 0.0288 & 0.0250 & 0.2630 \\
\hline
\end{tabular}

TABLE 6.4

Experimental convergence rates for the ASGS method according to the choice of the length scale in the stabilization parameters when $\nu=0$. Piecewise constant pressures.

\begin{tabular}{|c||c|c|c|c|}
\hline $\begin{array}{c}\text { Method } \\
\ell_{p}, \ell_{u}=\end{array}$ & $\begin{array}{c}\mathrm{A} \\
h, h\end{array}$ & $\begin{array}{c}\mathrm{B} \\
L_{0}, h\end{array}$ & $L_{0}{ }^{1 / 2} h^{1 / 2}, L_{0}{ }^{1 / 2} h^{1 / 2}$ & $L_{0}, L_{0}$ \\
\hline \hline$\left\|e_{u}\right\|$ & $-0.03(-)$ & $-0.01(-)$ & $0.86(1)$ & $1.90(1)$ \\
\hline$\left\|e_{p}\right\|$ & $-0.04(1)$ & $-0.03(-)$ & $0.96(1)$ & $1.84(1)$ \\
\hline$\left\|\nabla \cdot e_{u}\right\|$ & $-0.42(-)$ & $-0.03(-)$ & $0.55(-)$ & $1.56(1)$ \\
\hline
\end{tabular}

effective for this particular test. Using the OSS method we get similar results to those obtained for the ASGS method; let us remind that method B cannot be used in this case. From these results we can easily see that method $\mathrm{C}$ is the best choice. In Table 6.3 we have also included the norm of the different errors for the coarser mesh, using ASGS. For this mesh, method $\mathrm{C}$ is the most accurate for $\left\|\nabla e_{p}\right\|$, whereas method $\mathrm{D}$ is the most accurate for $\left\|\boldsymbol{e}_{u}\right\|,\left\|e_{p}\right\|$, and $\left\|\nabla \cdot \boldsymbol{e}_{u}\right\|$. In any case, method $\mathrm{C}$ is superior for finer meshes, because it exhibits a higher order of convergence.

We have carried out the same numerical experiments for discontinuous piecewise constant pressures. These results are included in Table 6.4 for the ASGS method and in Table 6.5 for the OSS method. In this case $k=l+1=1$. As expected, methods A and B (for the ASGS method) do not converge to the exact solution as the mesh size is reduced. From Theorems 4.1 and 4.2 we know that the improved error estimates obtained via duality for methods $\mathrm{A}$ and $\mathrm{B}$ (those with $\ell_{u}=h$ ) are only true for $c_{2}^{u}$ large enough. In our numerical experiments those orders of convergence are not fully observed, because the choice of $c_{2}^{u}$ does not satisfy this requirement. On the other hand, the results proved via duality for method $\mathrm{C}$ do not involve any assumption over 
TABLE 6.5

Experimental convergence rates for the OSS method according to the choice of the length scale in the stabilization parameters when $\nu=0$. Piecewise constant pressures.

\begin{tabular}{|c||c|c|c|}
\hline $\begin{array}{c}\text { Method } \\
\ell_{p}, \ell_{u}=\end{array}$ & $\begin{array}{c}\mathrm{A} \\
h, h\end{array}$ & $\mathrm{C}$ & $\mathrm{D}$ \\
${ }_{0}{ }^{1 / 2} h^{1 / 2}, L_{0}{ }^{1 / 2} h^{1 / 2}$ & $L_{0}, L_{0}$ \\
\hline \hline$\left\|e_{u}\right\|$ & $-0.03(-)$ & $0.87(1)$ & $1.87(1)$ \\
\hline$\left\|e_{p}\right\|$ & $-0.05(1)$ & $0.79(1)$ & $1.81(1)$ \\
\hline$\left\|\nabla \cdot e_{u}\right\|$ & $-0.40(-)$ & $0.54(-)$ & $1.55(1)$ \\
\hline
\end{tabular}

TABLE 6.6

Experimental convergence rates for the $O S S$ and $A S G S$ methods when $\sigma=0$. The P1/P1 pair.

\begin{tabular}{|c||c|c|}
\hline Method & ASGS & OSS \\
\hline \hline$\left\|e_{u}\right\|$ & $1.91(2)$ & $2.00(2)$ \\
\hline$\left\|e_{p}\right\|$ & $1.67(1)$ & $1.99(1)$ \\
\hline$\left\|\nabla \cdot e_{u}\right\|$ & $1.63(1)$ & $1.48(1)$ \\
\hline$\left\|\nabla e_{p}\right\|$ & $0.55(-)$ & $0.58(-)$ \\
\hline
\end{tabular}

TABLE 6.7

Experimental convergence rates for the OSS and ASGS methods when $\sigma=0$. Piecewise constant pressures.

\begin{tabular}{|c||c|c|}
\hline Method & ASGS & OSS \\
\hline \hline$\left\|\boldsymbol{e}_{u}\right\|$ & $1.98(2)$ & $1.98(2)$ \\
\hline$\left\|e_{p}\right\|$ & $1.45(1)$ & $1.42(1)$ \\
\hline$\left\|\nabla \cdot \boldsymbol{e}_{u}\right\|$ & $1.43(1)$ & $1.31(1)$ \\
\hline
\end{tabular}

this algorithmic constant, and the optimal orders of convergence have been reproduced by our numerical experiments. Method $\mathrm{C}$ converges to the good solution, but method $\mathrm{D}$ proves to be the right choice in this case. As inferred from the numerical analysis, method D is optimal for $k=l+1$.

6.2. Stokes flow. The second test deals with the limit case when $\sigma=0$, i.e., the Stokes problem. We consider the problem with exact solution:

$$
\boldsymbol{u}=(2 \pi \sin (2 \pi x) \cos (2 \pi y),-2 \pi \cos (2 \pi x) \cos (2 \pi y)), \quad p=\cos (2 \pi x) \cos (2 \pi y)-1
$$

We can easily check that the velocity is solenoidal, i.e., $g=0$. The regularity properties of the solution allow us to enforce the whole velocity on the boundary.

For the Stokes problem, all methods are equivalent because $\ell_{u}$ and $\ell_{p}$ are multiplied by $\sigma$ (equal to zero for Stokes flow) in the stabilization parameter. The experimental order of convergence using the ASGS and the OSS methods is shown in Table 6.6 for the P1/P1 interpolation spaces and in Table 6.7 when using discontinuous piecewise constant pressures. Both methods exhibit optimal convergence rates and superconvergence in some cases, like $\left\|e_{p}\right\|$ for the OSS method.

6.3. Stokes and Darcy flow coupled through interfaces. As a final numerical example, we consider a heterogeneous problem coupling Stokes and Darcy systems through an interface, as explained in section 5. We consider the problem 
TABLE 6.8

Experimental convergence rates for the ASGS method according to the choice of the length scale in the stabilization parameters for a heterogeneous domain coupling Stokes and Darcy subproblems. The $P 1 / P 1$ pair.

\begin{tabular}{|c||c|c|c|c|}
\hline $\begin{array}{c}\text { Method } \\
\ell_{p}, \ell_{u}=\end{array}$ & $\begin{array}{c}\mathrm{A} \\
h, h\end{array}$ & $\begin{array}{c}\mathrm{B} \\
L_{0}, h\end{array}$ & $L_{0}{ }^{1 / 2} h^{1 / 2}, L_{0}{ }^{1 / 2} h^{1 / 2}$ & $\mathrm{D}$ \\
$L_{0}, L_{0}$ \\
\hline \hline$\left\|e_{u}\right\|$ & $1.88(1)$ & $1.89(1)$ & $1.89(2)$ & $1.89(1)$ \\
\hline$\left\|e_{p}\right\|$ & $1.68(1)$ & $1.68(1)$ & $1.68(1)$ & $1.68(1)$ \\
\hline$\left\|\nabla \cdot e_{u}\right\|$ & $1.56(-)$ & $1.70(1)$ & $1.55(1)$ & $1.48(1)$ \\
\hline$\left\|\nabla e_{p}\right\|$ & $0.66(-)$ & $0.66(-)$ & $0.66(-)$ & $0.66(-)$ \\
\hline
\end{tabular}

TABLE 6.9

Experimental convergence rates for the OSS method according to the choice of the length scale in the stabilization parameters for a heterogeneous domain coupling Stokes and Darcy subproblems. The $P 1 / P 1$ pair.

\begin{tabular}{|c||c|c|c|}
\hline $\begin{array}{c}\text { Method } \\
\ell_{p}, \ell_{u}=\end{array}$ & $\begin{array}{c}\mathrm{A} \\
h, h\end{array}$ & $L_{0}{ }^{1 / 2} h^{1 / 2}, L_{0}{ }^{1 / 2} h^{1 / 2}$ & $\begin{array}{c}\mathrm{D} \\
L_{0}, L_{0}\end{array}$ \\
\hline \hline$\left\|\boldsymbol{e}_{u}\right\|$ & $1.95(1)$ & $2.17(2)$ & $2.05(1)$ \\
\hline$\left\|e_{p}\right\|$ & $1.77(1)$ & $1.77(1)$ & $1.77(1)$ \\
\hline$\left\|\nabla \cdot e_{u}\right\|$ & $0.99(-)$ & $2.15(1)$ & $2.36(1)$ \\
\hline$\left\|\nabla e_{p}\right\|$ & $0.58(-)$ & $0.58(-)$ & $0.58(-)$ \\
\hline
\end{tabular}

with exact solution:

$$
\boldsymbol{u}=(2 \pi \sin (2 \pi x) \cos (2 \pi y),-2 \pi \cos (2 \pi x) \cos (2 \pi y)), \quad p=\cos (2 \pi x) \cos (2 \pi y)-1,
$$

where $\boldsymbol{u}$ is equal to $\boldsymbol{u}^{S}$ on $\Omega^{S}$ and equal to $\boldsymbol{u}^{D}$ on $\Omega^{D}$. For this test, the tangential component of the normal stress for the Stokes problem vanishes on the interface; the Beavers-Joseph-Saffman condition (5.3) is reduced to a no-slip boundary condition. The whole velocity is enforced on $\partial \Omega^{S} \backslash \Sigma$, whereas only its normal component is constrained on $\partial \Omega^{D} \backslash \Sigma$. We use the formulation introduced in section 5 , and the coupled linear system is solved in a monolithic fashion (see (5.4)).

In Tables 6.8 and 6.9 we show the order of convergence for the $\mathrm{P} 1 / \mathrm{P} 1$ finite element pair using the ASGS and the OSS methods, respectively. It is clear from the experiments that the numerical error is driven by the Stokes problem in both cases. As a consequence, all methods exhibit a very similar behavior. The only exception is $\nabla \cdot \boldsymbol{e}_{u}$ for the OSS method that exhibits better convergence for methods $\mathrm{C}$ and D. Method $\mathrm{C}$ is particularly interesting for $k=l$ because it does not spoil the order of accuracy for the Stokes problem. Methods A, B, and D exhibit superconvergent behavior and do not spoil the accuracy of the Stokes problem for this particular case. However, these methods are not appealing in general, because the orders of convergence for Darcy flow are lower than those for Stokes flow.

The results for discontinuous pressures are provided in Tables 6.10 and 6.11. In this case, for methods A and B, the convergence for the Stokes problem is spoiled by the nonconvergence for the Darcy problem. As indicated for the pure Darcy problem, method D is optimal. Method C also converges to the exact solution, but more slowly. 
TABLE 6.10

Experimental convergence rates for the ASGS method according to the choice of the length scale in the stabilization parameters for a heterogeneous domain coupling Stokes and Darcy subproblems. Piecewise constant pressures.

\begin{tabular}{|c||c|c|c|c|}
\hline $\begin{array}{c}\text { Method } \\
\ell_{p}, \ell_{u}=\end{array}$ & $\begin{array}{c}\mathrm{A} \\
h, h\end{array}$ & $\begin{array}{c}\mathrm{B} \\
L_{0}, h\end{array}$ & $L_{0}{ }^{1 / 2} h^{1 / 2}, L_{0}{ }^{1 / 2} h^{1 / 2}$ & $\begin{array}{c}\mathrm{D} \\
L_{0}, L_{0}\end{array}$ \\
\hline \hline$\left\|e_{u}\right\|$ & $-0.03(-)$ & $-0.02(-)$ & $0.86(1)$ & $1.94(1)$ \\
\hline$\left\|e_{p}\right\|$ & $0.26(1)$ & $0.29(-)$ & $1.48(1)$ & $1.70(1)$ \\
\hline$\left\|\nabla \cdot \boldsymbol{e}_{u}\right\|$ & $-0.46(-)$ & $-0.02(-)$ & $0.67(-)$ & $1.72(1)$ \\
\hline
\end{tabular}

\section{TABLE 6.11}

Experimental convergence rates for the OSS method according to the choice of the length scale in the stabilization parameters for a heterogeneous domain coupling Stokes and Darcy subproblems. Piecewise constant pressures.

\begin{tabular}{|c||c|c|c|}
\hline $\begin{array}{c}\text { Method } \\
\ell_{p}, \ell_{u}=\end{array}$ & $\mathrm{A}$ & $\mathrm{C}$ & $\mathrm{D}$ \\
\hline \hline$\left\|\boldsymbol{e}_{u}\right\|$ & $-0.02(-)$ & $0.90(1)$ & $1.95(1)$ \\
\hline$\left\|e_{p}\right\|$ & $0.84(1)$ & $1.64(1)$ & $1.68(1)$ \\
\hline$\left\|\nabla \cdot \boldsymbol{e}_{u}\right\|$ & $-0.17(-)$ & $0.68(-)$ & $1.72(1)$ \\
\hline
\end{tabular}

7. Conclusions. Two stabilized finite element formulations have been proposed in this paper to deal with the Stokes-Darcy problem, namely, the ASGS formulation and the OSS method. A major contribution of this work is to provide a unified analysis encompassing the cases $\nu=0$ (Darcy's problem) and $\sigma=0$ (Stokes' problem). To this end, the key point is to work with a norm that contains all the terms for which stability can be proved (provided the data are regular enough).

For the finite element problems, it has been shown that the convergence properties of the method in the Darcy limit $(\nu=0)$ are very much influenced by the choice of the length scales that appear in the stabilization parameters. From the practical point of view, it is often claimed that equal velocity-pressure interpolation is the most effective choice to implement. In this case, our analysis reveals that the length scales have to be chosen as $\left(L_{0} h\right)^{1 / 2}$, where $L_{0}$ is a characteristic length of the domain and $h$ the mesh size of the finite element discretization.

With regard to the Darcy problem, the choice of the method does depend on the order of approximation of velocities and pressures. Depending on that, we have drawn some recommendations. It is important to note that we have developed the first stabilized method for Darcy flow that accommodates piecewise constant pressures, taking the length scale as $L_{0}$.

Numerical experiments have confirmed the theoretical rates of convergence, including those obtained through duality arguments. Optimal convergence rates have been found for the Stokes-Darcy problem, for the Darcy problem, and for the Stokes and the Darcy problems solved in different subdomains and coupled through a common interface.

\section{REFERENCES}

[1] T. Arbogast and D.S. Brunson, A computatonal method for approximating a Darcy-Stokes system governing a vuggy porous medium, Comput. Geosci., 11 (2007), pp. 207-218.

[2] S. BADia AND R. Codina, Stabilized continuous and discontinuous Galerkin techniques for Darcy flow, UPCommons, http://hdl.handle.net/2117/2447, submitted. 
[3] G.S. Beavers and D.D. Joseph, Boundary conditions at a naturally permeable wall, J. Fluid Mech., 30 (1967), pp. 197-207.

[4] P. Bochev And C.R. Dohrmann, A computational study of stabilized, low-order $C^{0}$ finite element approximations of Darcy equations, Comput. Mech., 38 (2006), pp. 323-333.

[5] P. Bochev and M. Gunzburguer, A locally conservative least-squares method for Darcy flows, Comm. Num. Methods Engrg., 24 (2008), pp. 97-110.

[6] F. Brezzi And M. Fortin, Mixed and Hybrid Finite Element Methods, Springer-Verlag, Berlin, 1991.

[7] F. Brezzi, T.J.R. Hughes, L.D. Marini, And A. Masud, Mixed discontinuous Galerkin methods for Darcy flow, J. Sci. Comput., 22-23 (2005), pp. 119-145.

[8] F. Brezzi, T.J.R. Hughes, And E. SüLI, Variational approximation of flux in conforming finite element methods for elliptic partial differential equations: A model problem, Rend. Lincei Mat. Appl., 12 (2001), pp. 167-183.

[9] E. Burman and P. Hansbo, A unified stabilized method for Stokes' and Darcy's equations, J. Comput. Appl. Math., 198 (2007), pp. 35-51.

[10] R. CodinA, Stabilization of incompressibility and convection through orthogonal sub-scales in finite element methods, Comput. Methods Appl. Mech. Engrg., 190 (2000), pp. 1579-1599.

[11] R. Codina, A stabilized finite element method for generalized stationary incompressible flows, Comput. Methods Appl. Mech. Engrg., 190 (2001), pp. 2681-2706.

[12] R. CodinA, Stabilized finite element approximation of transient incompressible flows using orthogonal subscales, Comput. Methods Appl. Mech. Engrg, 191 (2002), pp. 4295-4321.

[13] R. Codina, Analysis of a stabilized finite element approximation of the Oseen equations using orthogonal subscales, Appl. Numer. Math., 58 (2008), pp. 264-283.

[14] R. Codina, Finite element approximation of the three-field formulation of the Stokes problem using arbitrary interpolations, SIAM J. Numer. Anal., 47 (2009), pp. 699-718.

[15] R. Codina, J. Principe, And J. Baiges, Subscales on the element boundaries in the variational two-scale finite element method, Comput. Methods Appl. Mech. Engrg., 198 (2009), pp 838-852.

[16] M. Discacciati, E. Miglio, And A. Quarteroni, Mathematical and numerical models for coupling surface and groundwater flows, Appl. Numer. Math., 43 (2002), pp. 57-74.

[17] M. Discacciati, A. Quarteroni, And A. Valli, Robin-Robin domain decomposition methods for the Stokes-Darcy coupling, SIAM J. Numer. Anal., 45 (2007), pp. 1246-1268.

[18] C.R. Dohrmann And P.B. Bochev, A stabilized finite element method for the Stokes problem based on polynomial pressure projections, Int. J. Numer. Methods Fluids, 46 (2004), pp. 183-201.

[19] A. Ern and J.-L. Guermond, Theory and Practice of Finite Elements, Springer-Verlag, Berlin, 2004.

[20] T.J.R. Hughes, Multiscale phenomena: Green's function, the Dirichlet-to-Neumann formulation, subgrid scale models, bubbles and the origins of stabilized formulations, Comput. Methods Appl. Mech. Engrg., 127 (1995), pp. 387-401.

[21] T.J.R. Hughes, G.R. FeiJóo, L. Mazzei, And J.B. Quincy, The variational multiscale method-a paradigm for computational mechanics, Comput. Methods Appl. Mech. Engrg., 166 (1998), pp. 3-24.

[22] T.J.R. Hughes, A. Masud, And J. WAn, A stabilized mixed discontinuous Galerkin method for Darcy flow, Comput. Methods Appl. Mech. Engrg., 195 (2006), pp. 3347-3381.

[23] W.J. Layton, F. Schieweck, And I. Yotov, Coupling fluid flow with porous media flow, SIAM J. Numer. Anal., 40 (2002), pp. 2195-2218.

[24] K.A. Mardal, X.C. Tai, And R. Winther, A robust finite element method for Darcy-Stokes flow, SIAM J. Numer. Anal., 40 (2002), pp. 1605-1631.

[25] A. Masud and T.J.R. Hughes, A stabilized mixed finite element method for Darcy flow, Comput. Methods Appl. Mech. Engrg., 191 (2002), pp. 4341-4370.

[26] K.B. Nakshatrala, D.Z. Turner, K.D. Huelmstad, and A. Masud, A stabilized mixed finite element method for Darcy flow based on a multiscale decomposition of the solution, Comput. Methods Appl. Mech. Engrg., 195 (2006), pp. 4036-4049.

[27] B. Rivière And I. Yotov, Locally conservative coupling of Stokes and Darcy flows, SIAM J. Numer. Anal., 42 (2005), pp. 1959-1977.

[28] P. Saffman, On the boundary condition at the surface of a porous medium, Stud. Appl. Math., 1 (1971), pp. 93-101.

Copyright (c) by SIAM. Unauthorized reproduction of this article is prohibited. 